\title{
Galactic Archaeology. The dwarfs that survived and perished
}

\author{
Vasily Belokurov ${ }^{\mathrm{a}, *}$ \\ ${ }^{a}$ Institute of Astronomy, Cambridge
}

\begin{abstract}
From the archaeological point of view, the local dwarf galaxies are unique objects in which the imprint of the conditions that shaped the early structure formation can be studied today at high resolution. Over the last decade, this new window into the high redshift Universe has started to be exploited using deep wide-field imaging, high resolution spectroscopy and cutting edge Nbody and hydro-dynamical simulations. We review the recent advances in the observational studies of the Milky Way dwarf galaxies, with the aim to understand the properties of the population as a whole and to assist an objective comparison between the models and the data.
\end{abstract}

Keywords:

Galaxies: kinematics and dynamics, Galaxies: dwarf, dark matter, Local Group, Galaxies: stellar content.

\section{Introduction}

The prehistoric stars whose formation epochs lie beyond the redshift accessible by the Hubble Ultra Deep Field, have been found en masse in little satellites around the Milky Way. Other less fortunate dwarf galaxies have been pulled apart by gravity to furnish the diffuse Galactic halo. These recently uncovered relics of the ancient dwarf galaxy population may play a vital role in the pursuit of reconstructing the formation of the Galaxy. Its path from the distant pre-reionisation era, through the most active growth

\footnotetext{
${ }^{*}$ Corresponding author

Email address: vasily@ast.cam.ac.uk (Vasily Belokurov)
} 
periods to the present day, can be gleaned by studying the chemical composition and the phase-space density distribution of these halo denizens. How this Galactic archaeological record is collected and analyzed, and what aspects of galaxy (dwarf and otherwise) formation and evolution it illuminates is the topic of this review.

The field of Galactic and Local Group studies has enjoyed a decade of unprecedented busyness thanks to the abundance of data supplied by several generations of wide-angle sky surveys and the coming of age of N-body and hydro-dynamic computer simulations of galaxy formation. Accordingly, there have been several fresh in-depth reviews of the matters relevant to the topic of this article. In particular, the star-formation histories and abundances of the Milky Way dwarf galaxies have been scrutinized by Tolstoy et al. (2009). McConnachie (2012) has painstakingly assembled a homogenized database of the properties of all known dwarfs within $3 \mathrm{Mpc}$. Walker (2012) has written down a scrupulous account of the current evidence for the presence of Dark Matter in dwarfs, while Kravtsov (2010) has reviewed the progress in reconciling the mismatch in the appearance of the real dwarf satellites and the toy ones built into simulated Dark Matter sub-structure. The quest for the least luminous galaxies, so-called ultra-faint dwarfs, has been documented by Willman (2010). To complement these, a review of the structure, chemistry and dynamics of the Galactic stellar halo can be found in Helmi (2008). Finally, Ivezić et al. (2012) explain exactly how the large surveys like the SDSS have revolutionized the way research into the Galactic stellar populations is conducted.

This review will attempt to avoid boring the reader with re-stating the facts already discussed thoroughly in the works above. Instead, its purpose is to report on the most recent progress in the area of the Galactic Archaeology and to list some of the burning questions that are destined to be answered with the upcoming sky surveys.

\section{Little galaxies. Big questions.}

The main premise of the current galaxy evolution theory, which itself exists within the broader theory of the Universal structure formation a.k.a. $\Lambda \mathrm{CDM}$, postulates that all galaxies are born, live and die inside dark matter (DM) halos. $\Lambda$ CDM uses Cold Dark Matter to provide nucleation sites for the subsequent budding of galaxies of all sizes. Small lumps in the primordial DM gravy are the most numerous and develop the quickest, therefore the first 
baryonic systems to appear are the dwarf galaxies. What happens later is the competition between the expansion of the Universe and the gravitational pull of the emerging DM halos. In this game, the majority of the dwarfs eventually lose: they are dragged into deeper potential wells, where they get undone and their matter, dark and otherwise, is subsumed to form bigger galaxies.

A small fraction of the aboriginal dwarf satellite population that survives the tidal disruption during the accretion should, in principle, be detectable in and around the Galaxy today. $\Lambda \mathrm{CDM}$ is a young theory and it is perfectly reasonable that its fundamental properties are still being actively questioned. One key prediction of the theory is the existence of the large number of small DM halos (called sub-halos to indicate the hierarchical nature of all structures in this paradigm) in a galaxy like our own. The theory then postulates that dwarf satellites are the sub-halos that have accreted enough gas and have held on to it long enough to cool it down and form stars. Those dwarfs that have survived until $z=0$ must have either stayed well away from the strong tides of the central Milky Way or are just arriving to their final destination (see e.g. Bullock and Johnston, 2005).

\subsection{Galactic dwarf census}

Galactic Archaeology provides the observational evidence of the accretion onto the Milky Way and the statistics of the survived and the destroyed dwarfs. This data can be interpreted within the current hierarchical structure formation model provided the processes to do with star formation and interaction between the baryons and the DM are well understood. For example, Klypin et al. (1999) demonstrate how merely the count of the Galactic dwarf galaxies can be used to challenge the very foundations of the accepted galaxy formation paradigm. As they show, it is possible that to reconcile the measurement and the prediction of the satellite mass function, a cut-off in the power spectrum of the primordial matter fluctuations on small scales is required. Alternatively, they point out, it is perfectly feasible that the suppression of star formation in low-mass systems is stronger than expected, thus leaving the vast majority of sub-halos dark forever.

The number of the low-mass galaxies around the Milky Way has more than doubled in the last ten years solely due to the supply of high quality all-sky data from the SDSS. Yet, any attempt to corroborate the theory of dwarf formation based on these recent discoveries, would be hopeless without first quantifying how much the rapidly growing satellite sample is influenced 
by the selection effects. All Galactic dwarfs (excluding the Sagittarius and the Sextans dSphs) discovered before the first SDSS data releases and now conventionally known as Classical were found by simply eye-balling the photographic plates. The SDSS has changed the game completely: today the science-ready catalogs containing hundreds of millions of stars and galaxies can be trawled through quickly, making the search efficient and quantifiable. Using the early released data from only two SDSS runs, Willman et al. (2002) estimates the sensitivity of the full survey dataset to resolved Galactic companions and predicts that the satellite Luminosity Function can be straightforwardly constructed facilitating the first unbiased comparison between the theory and observations. Accordingly, Koposov et al. (2008) present the completeness calculation for the SDSS DR5 based on the fullyautomated satellite detection algorithm and a large suite of realistic mock observations of dwarf galaxies of various sizes and luminosities. The results presented by Koposov et al. (2008) show clearly that there are large swathes of the parameter space where the Milky Way satellites can be detected with nearly $100 \%$ efficiency. Outside these regions, the detectability drops sharply to 0 . The transition boundary is controlled by the surface brightness limit of the SDSS $\mu_{\mathrm{lim}}$, the luminosity of the satellite and its distance. Alternatively, for all objects with $\mu_{\text {lim }}<30$ mag $\operatorname{arcsec}^{-2}$, the SDSS completeness can be expressed in terms of the volume within which it discovered all satellites of a particular luminosity.

According to Koposov et al. (2008), the sample of dwarf satellites with luminosities brighter than $M_{V} \sim-5$ is essentially complete out to the Galactic virial radius $r_{\text {vir }}=280 \mathrm{kpc}$ within the SDSS DR5 field of view. However, the accessible volume plummets fast with decreasing dwarf luminosity, which means that for satellites as faint as Segue 1 with $M_{V} \sim-3$, only few percent of the virial volume have been probed. Importantly, using thus-calculated fraction of the total Galactic volume sampled, it is now possible to predict the complete number of the satellites if their distribution with radius is assumed. Naturally, the flatter the radial distribution of the satellites the bigger is faction of objects remaining to be detected. Koposov et al. (2008) surmise that if the number of dwarfs decays in a NFW-like fashion $\left(\propto r^{-3}\right.$ at large distances) then the Luminosity Function (LF) of the Milky Way satellites goes as $\sim 10^{0.1\left(M_{V}+5\right)}$. According to this LF, the objects with luminosities between $M_{V}=-2$ and $M_{V}=-5$ contribute just under a half of the total $\sim 100$ dwarfs. Some 4 times more satellites is predicted by Tollerud et al. (2008) who take advantage of the completeness calculation published by Ko- 


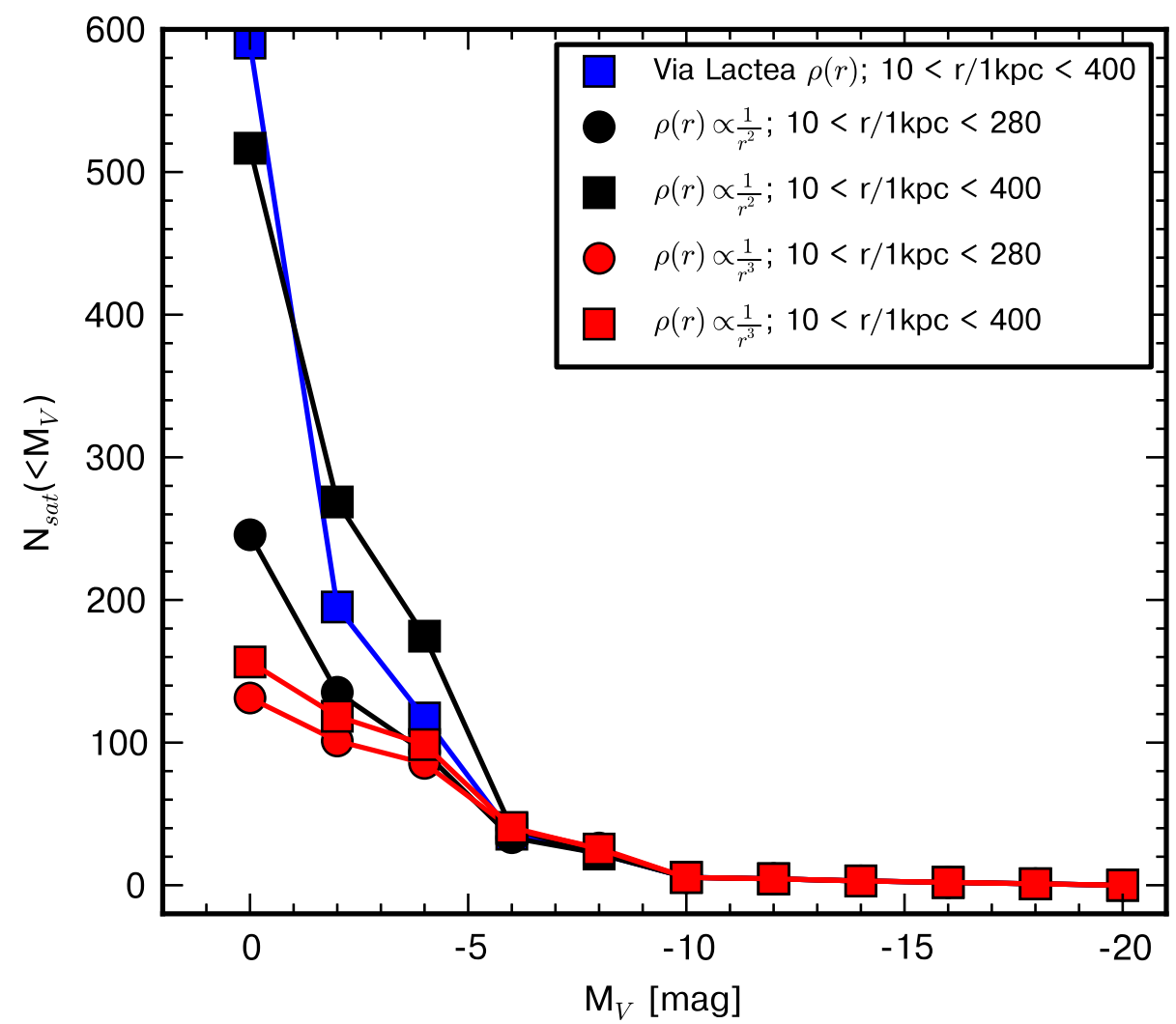

Figure 1: Dependence of the Galactic dwarf luminosity function on the assumed radial density profile of the satellites. This shows the cumulative luminosity functions (the total number of objects as a function of their absolute magnitude) for the Milky Way satellites in the spherical volume with radius $R=280 \mathrm{kpc}$ (filled circles), which is similar to the volume used in Koposov et al. (2008); and with $R=400 \mathrm{kpc}$ (squares), similar to the maximal distance used in Tollerud et al. (2008). Red curves give the LFs obtained with the satellite radial profile proportional to $r^{-3}$, while the LFs given in black assume that the number of satellites decays as $r^{-2}$. Blue curve is the LF based on the radial distribution of the Via Lactea sub-halos. All density laws are truncated at $R=10 \mathrm{kpc}$, the satellite detection efficiency applied is from Koposov et al. (2008). Only $\sim 100$ dwarfs are predicted to inhabit the Galaxy if their radial number density follows the $r^{-3}$ law (red curves), i.e. a NFW distribution with small scale radius, see also Koposov et al. (2008). Substantially more ultra-faint objects are anticipated if the inverse square law is adopted, particularly if the dwarf population extends to distances as large as $R=400 \mathrm{kpc}$ (black squares). In Via Lactea simulation, in the inner $60-80 \mathrm{kpc}$, the number density of sub-halos is even flatter as shown in the Supplementary Figure 1 of Diemand et al. (2008). Accordingly, most of the faintest satellites are supposed to be undetected by the SDSS, as reflected in the quick rise of the LF (in blue) at $M_{V} \sim-3$, see also Tollerud et al. (2008). Figure courtesy of Sergey Koposov, IoA. 
posov et al. (2008), but choose to adopt the radial distribution of sub-halos from the Via Lactea N-body simulation (Diemand et al., 2008). In the inner $60-80 \mathrm{kpc}$, the distribution of sub-halos in Via Lactea drops very slowly with radius. In fact, for several tens of $\mathrm{kpc}$ it is almost flat according to the Supplementary Figure 1 of Diemand et al. (2008). The effect of this large "core" in the satellite number density profile on the dwarf LF is particularly striking for the faintest of the satellites, those detectable by the SDSS only out to $30-40 \mathrm{kpc}$. Out of the fiducial $\sim 400$ satellites predicted by Tollerud et al. (2008), almost $90 \%$ are those with $-2<M_{V}<-5$. The drastic dependence of the Galactic dwarf LF on the assumed radial distribution of satellites is illustrated in Figure 1. From these first attempts to gauge the size of the Galactic satellite body, the important role played by the faintest of the dwarfs emerges.

\subsection{Unexplored variety of hierarchical galaxy formation}

In the decade following the publication of the two whistle-blowing papers by Klypin et al. (1999) and Moore et al. (1999), the sub-halo abundance matching (SHAM) technique that relies on assigning higher stellar luminosities to the DM sub-halos with higher maximal masses, has been optimized near to perfection. The success of the abundance matching has created a brief period of the comforting lull with the gaping void between dwarf galaxies and sub-halos (known as the missing satellites problem) seemingly breached (e.g. Koposov et al., 2009, Macciò et al., 2010). However, pitted against the existent Galactic satellites, these mock dwarfs do not stand a close scrutiny: the density profiles in the most massive systems are not corroborated by the available kinematics (see e.g. Boylan-Kolchin et al., 2012). It turns out, the overall star-formation efficiency is now such a strong function of the sub-halo mass that the dwarfs with modest stellar masses and velocity dispersions are forced to inhabit disproportionally massive and dense halos. What is missing from this picture drawn with the help of SHAM? It appears quite a few of the vital ingredients might be lacking.

To begin with, the influence of the rather significant baryonic disk in the Galaxy has been surprisingly overlooked in many of the SHAM studies. Yet, as demonstrated in a recent succession of papers (e.g. Taylor and Babul, 2001; Read et al., 2006; Peñarrubia et al., 2010; D'Onghia et al., 2010), disks aide the tidal destruction of satellites thus seriously depleting the number

of the dwarf survivors. One crucial detail is noted by Peñarrubia et al. (2010): dwarf galaxies with cored density profiles are less likely to survive 
the devastating action of the disk. $\Lambda \mathrm{CDM}$ on its own does not permit cored dark matter profiles, but Read et al. (2006) and Pontzen and Governato (2012) show that the gas flow due to the persistent supernova feedback can pull dark matter along to the outskirts of dwarf galaxies. Accordingly, a plausible setup in which the satellite survival rate at $z=0$ is regulated by the interplay between the strong stellar feedback evacuating the centers of sub-halos and the enhanced tidal disruption due to the disk is described in the work by Brooks et al. (2013). Here, by means of applying a simple correction to the central masses of semi-analytical dwarfs, as originally proposed by Zolotov et al. (2012), many of the massive satellite galaxies are wrecked, and the tension between the data and the theory seems to be alleviated once again. Note, however, that the total amounts of supernova energy required to cause appreciable damage to the DM central density cusps have been deemed excessive by many authors (e.g. Peñarrubia et al., 2012; GarrisonKimmel et al., 2013).

As the physics of star formation is just starting to be explored, there does not exist a single hydro-dynamical simulation of the Milky Way run at the resolution appropriate to resolve the gas infall and cooling at all epochs from high redshifts to the present day. Instead, on a star by star basis, the processes that play the most important role (like cooling and feedback) are gleaned, synopsized and subsequently incorporated into the simulations as sub-grid recipes to be followed together with the laws of the Newtonian gravity (and sometimes hydrodynamics). In this approach, it is believed that, the evolution of the dark matter density on the relevant scales has been fully captured with the latest pure N-body simulations. However, as the DM particles are followed from the distant past to the current day, the actual sequence of accretion events the Milky Way prototype goes through varies considerably from host to host. This has profound implications on the final shape of the DM sub-halo mass function (MF), and ultimately on the properties of the Galaxy's dwarf satellite population.

\subsubsection{Host mass and concentration}

When faced with the myriad of DM halos to furnish a Milky Way together with its satellites, the conventional choice is to select the host by matching its virial mass to that of the Galaxy. Note that according to Figure 8 in Springel et al. (2008), in the 6 host halos with different virial masses, the sub-halo number counts in the bins of mass scaled to the the mass of the host lie exactly on top of each other. Accordingly, it is established that the 
relative MF scales as $\left(M_{\text {sub }} / M_{50}\right)^{-1.9}$ and the absolute sub-halo abundance normalization includes another factor of $M_{50}$ for the host mass. Thus, for the hosts whose masses are different by $100 \%$ the total sub-halo counts would also disagree by a factor of 2. Alas, the mass of the real Milky Way is not known with the accuracy as high as $100 \%$. Even though, depending on the method used, the formal uncertainties can be as low as 30\%, there are serious disagreements between the measurements making the systematic error much higher. For example, according to Watkins et al. (2010), the plausible range for the Milky Way mass within $300 \mathrm{kpc}$ is approximately from $1 \times 10^{12} M_{\odot}$ to $3 \times 10^{12} M_{\odot}$, which would imply a factor of $\sim 3$ difference in the total number of sub-halos.

The present uncertainty in the measurements of the Milky Way's concentration is $\sim 100 \%$, which is perhaps even more appalling since the allowed range of concentrations is much smaller. The concentration $c=r_{v i r} / r_{s}$ of a DM halo describes how dense its inner parts (within the scale radius $r_{s}$ ) are compared to the halo overall (out to the virial radius $r_{\text {vir }}$ ). For the halos of similar virial mass, their concentrations are ultimately linked to the shape of the host's mass assembly history, with those peaking at very early times producing higher concentrations (see e.g. Wechsler et al., 2002). For the population of dwarf satellites at redshift zero, having a host halo with a high concentration is a double whammy. The first implication is obvious: if the accretion history settled into the quiescent phase at high redshifts, at later times, fewer dwarfs will be accreted. Second, having a dense pile up of dark matter (and baryons) means more efficient tidal disruption and the lower satellite survival rate. According to the N-body simulations, for the halos with Milky Way-like masses, the concentration is predicted to be of order of $c=12 \pm 3$ (see e.g. Boylan-Kolchin et al., 2010).

There are four techniques available today to measure the Galactic mass, each with its own assumptions and inherent limitations. The first three rely on the kinematics of a sample of the gravitational potential tracers, and therefore can only be applied straightforwardly within few tenths of the Galactic virial radius. The first method uses stars or gas to determine the run of the Galactic rotation velocity $v_{\text {rot }}=\sqrt{r \frac{\mathrm{d} \Phi}{\mathrm{d} r}}$ (where $\Phi$ is the underlying potential) with Galacto-centric radius $r$. Naturally, the rotation curve can only be sampled within $r<20 \mathrm{kpc}$, as there is no indication that the disk continues much beyond that. Having the full $6 \mathrm{D}$ information is rare, the latest such attempt presents the measurements of trigonometric parallaxes 
for a dozen or so masers, from which the circular rotation speed at the Sun's location is deduced (Reid et al., 2009). Alternatively, Bovy et al. (2012) shows that the circular velocity can be inferred by marginalizing over poorly constrained distances and unknown proper motions for a large $(>3,000)$ set of (mostly) disk giant stars with accurate line-of-sight velocities.

An equally rewarding, but perhaps yet a more challenging approach is to gauge the Galactic escape speed $v_{\text {esc }}=\sqrt{2|\Phi|}$ by analyzing the tail of the stellar velocity distribution. The results are sensitive to the quality of the distance and the proper motion data, in particular, imperfect proper motion measurements are so detrimental that, normally, they are avoided altogether. Instead, a velocity distribution function is chosen, whose exact shape is controlled by a small number of parameters that get simultaneously constrained in the process of the likelihood maximization. For example, using a relatively small sample (16) of high-velocity stars provided by the earlier releases of the RAVE survey, Smith et al. (2007) measure the local escape speed. Conveniently, given the Galactic escape speed and assuming the contributions of the bulge and the disk to the total potential, the mass and the concentration of the Milky Way's halo also can be extracted. The analysis by Smith et al. (2007) seems to prefer the Galaxy with the mass as low as $0.9 \times 10^{12} M_{\odot}$ and the concentration as high as 24 . While the applicability of both the circular speed and the escape speed techniques is restricted to the inner Galaxy, the latter has the advantage of probing the Galactic mass out of the disk plane.

Most of the Milky Way's mass lies beyond the extent of the disk, hence at large Galacto-centric distances, a different approach is required. Given enough mass tracers (stars or satellites) in a wide range of locations throughout the Galaxy, the total mass profile can be obtained by means of Jeans modelling of the tracer kinematics (see e.g. Battaglia et al., 2005). The terms that enter the spherical Jeans equation are: the tracer density, the tracer velocity dispersion and the tracer velocity anisotropy. At large distances, only one of these might be available, namely the line-of-sight velocity dispersion. Making the Jeans analysis of the far reaches of the Galactic halo possible clearly falls within the realm of Galactic Archaeology which can both deliver the most distant tracers as well as constrain the overall tracer density distribution. The stumbling block, however, is the scarcity of tracers with the tangential components of the velocity measured. As a consequence, the anistropy is normally treated as a nuisance parameter since the most datasets available lack in accuracy and breadth to constrain it. Even with the arrival of Gaia, 
the situation will only improve for the nearby objects, leaving the distant ones wanting in more precise proper motions. While assigning anistropy to a tracer population is a solution far from ideal, presently, it is the Jeans modelling together with its variants that provides the most stringent constraints on the total mass of the Milky Way (e.g. Xue et al., 2008).

Finally, a new, conceptually different method to probe the matter distribution in the Galaxy is now coming of age. Compared to the three approaches discussed above, it does not rely at all on the instantaneous kinematic properties of large samples of tracers, and thus, for example, needs no assumption of their velocity anisotropy. Stellar tidal streams are shown to align closely with the obit of their disrupting (or disrupted) progenitor and therefore give an almost direct way of measuring the underlying potential. Recently, the power of the method has been demonstrated beautifully by Koposov et al. (2010) who, using the 6D data of the GD-1 stream, measured the Galactic rotation curve locally. This type of analysis can, in principle, be extended to distances beyond the predicted Galaxy's scale radius $r_{s}$. The prime source of degeneracy in recovering the Galactic potential using tidal tails, is the length of the stream available. However, to date, for several distant streams there exists sufficient data covering tens (Orphan Stream with the maximal distance of $\sim 50 \mathrm{kpc}$ Belokurov et al., 2007b; Newberg et al., 2010) or even hundreds of degrees (Sagittarius Stream with the maximal distance of $\sim 100$ kpc, e.g. Majewski et al., 2004; Newberg et al., 2003; Belokurov et al., 2006a; Yanny et al., 2009; Belokurov et al., 2013). Given the magnitude limit of the on-going imaging surveys like SDSS or Pan-STARRS, for stellar streams to be detected so far out in the halo, the progenitor's luminosity, and therefore mass, ought to be substantial. This bias implies that the currently known distant streams can not be appropriately modeled using simple orbit approximation, the circumstance that now can be mitigated with the arrival of more sophisticated modeling techniques (e.g. Eyre and Binney, 2011; Sanders and Binney, 2013)

\subsubsection{Mass assembly history and environment}

The computational expense of running numerical simulations of Galactic halos at the resolution adequate to capture the properties of the halo substructure is prohibitively high. Hence, the comparison between DM sub-halos and the observed dwarfs has been based on the analysis of only 8 N-body simulations: a sample of 6 Aquarius halos (Springel et al., 2008), complemented by halos of Via Lactea II (Diemand et al., 2008) and GHalo (Stadel 
et al., 2009). For this reason, the host-to-host variation of the dark and the luminous sub-structure remains largely un-studied. As well as improving the resolution and the speed of the simulations, there is an ongoing effort to quantify the complex diversity of structures forming within $\Lambda$ CDM with a handful of key parameters, e.g. host halo mass, shape of the accretion history and significance of the overdensity of the local volume. These, of course, are inter-related: the mass of the DM halo hosting a Milky Way galaxy at redshift $z=0$ is the sum total over its accretion history, which in turn is dictated by the whereabouts of the halo within the cosmic Large Scale Structure. While the importance of not knowing such an elementary property of the Galaxy like its mass is now accepted, the impact of the location of the Milky Way within the larger cosmic structure and the details of its accretion history are just beginning to be investigated.

Today, there exist two intriguing constraints on the Milky Way's accretion history. First is the observation that the Galactic disk probably has to survive intact for some 7-10 Gyr (e.g. Figure 18 of Burnett et al., 2011). This, therefore, potentially excludes any significant mergers between $z \sim 1$ and now. Second is the new observational and numerical evidence for the late infall of the Magellanic Clouds (e.g. Besla et al., 2010). This signifies the end of the quiescent phase in the Galactic accretion history and can be exploited to place useful constraints on the mass assembly of the Galaxy (e.g. Busha et al., 2011a). What happened before the quiescent phase, why did it begin and why did it end? How common is this particular shape of the mass assembly history (MAH) amongst other disk galaxies of similar mass? Was the early accretion dominated by small satellite infall and was it synchronized? Or perhaps, was the bulk of the Galactic matter instead acquired in one or two mergers with massive nearby fragments? Unfortunately, these questions remain largely unanswered and therefore, a variety of loose ends continues to confuse the current picture of the Galaxy formation and muddle the modelling of the nearby dwarfs. For example, if many small satellites are accreted early on, enough should survive and be detectable today. On the contrary, massive mergers usually lead to an entirely different outcome: in this case, the dynamical friction is strong enough to slow the dwarf down thus boosting its plunge into the inner Galaxy where it is quickly disrupted. These two scenarios can be identical in terms of the epoch of accretion and the total mass accreted, yet they can produce dramatically different dwarf satellite populations at $z=0$.

An attempt to quantify the amplitude of the host-to-host scatter in the 
properties of artificial Galactic dwarfs using analytic models is presented in Purcell and Zentner (2012). The piece-de-résistance of the method is the Monte-Carlo sampling of an arbitrary large number of different accretion histories (as described in Zentner et al., 2005a). Using this technique, it can be demonstrated that the scatter in the possible MAHs is naturally large enough for the Milky Way-like halo to host a satellite population consistent with the observed one in 10\%-20\% of cases. These results, within the limitations of the method, shed light onto the statistical significance of the "too-big-to-fail" problem (Boylan-Kolchin et al., 2012): there does not have to be a serious excess of massive invisible sub-halos in the Galaxy. Interestingly, together with the recently invoked lower Galaxy mass (e.g. Vera-Ciro et al., 2013) and the strong stellar feedback (e.g. Brooks et al., 2013), this is now the third solution for the potential problem identified by Boylan-Kolchin et al. (2012). It would seem that if all three methods are as efficient as described, there could be very few satellites left around the Galaxy! It is, therefore, the most urgent task for the Galactic Archaeology to provide new observational constraints of the Milky Way's accretion history through studies of the spatial and the chemo-dynamical distributions of the ancient stellar halo populations.

The Milky Way is not a solitary field spiral: together with its neighbor of approximately the same mass, Andromeda and its satellites, it makes up the small slightly over-dense region of the Universe known as the Local Group of galaxies. The so-called assembly bias stipulates an excess of probability of finding a massive satellite sub-halo around hosts situated in higher density regions as compared to those in under-dense environments (e.g. Wechsler et al., 2006). Possibly, this effect could go some way to explaining the presence around the Milky Way satellites as massive as the Magellanic Clouds. According to Busha et al. (2011b), while for the field halo of Milky Way-like mass, the probability to host LMC/SMC pair is of order of 5\%-11\%, having another host halo of similar mass in the vicinity boosts it up to $25 \%$. This is good news, but are these sub-halos on their first (or perhaps second) passage around the simulated Galaxies as the Milky Way observations seem to indicate? A unique investigation is described in Forero-Romero et al. (2011) who use a suite of so-called constrained simulations of the Local Group (CLUES, see http://www.clues-project.org/) in which the broad-brush features of the Milky Way-Andromeda pair are reproduced, to study the assembly history of either host halo. They find that i) both galaxies had their last significant accretion event some 10-12 Gyr ago, and that ii) this particular common accretion history is quite rare (from $1 \%$ to $3 \%$ ) amongst the pairs of host 
halos in Bolshoi simulation. This conclusion appears to be in contradiction with the studies in which the Clouds are just being accreted.

\subsection{Tidal origin of the local dwarf galaxies}

It is inspiriting that there exists at least one alternative, and, importantly, testable scenario of the formation of dwarf satellites in and around the Milky Way. Lynden-Bell (1976) first pointed out the proximity of the several of the Galactic dwarfs to the LMC's orbital plane as defined by the gaseous stream leading the Cloud. The hypothesis then put forward is of a Greater Magellanic Galaxy that had been torn apart as it interacted with the Milky Way, giving birth to the Large and Small Clouds, as well as to a litter of smaller dwarfs. A quarter of a century later, with the measurement of the space velocities of the satellites in hand, the surprising juxtaposition of the orbital planes of the LMC, SMC, UMi and Dra is confirmed (e.g. Palma et al., 2002). This motivates Kroupa et al. (2005) to claim that the observed distribution of the Galactic satellites is too anisotropic to fit seamlessly within the CDM paradigm. In the authors' opinion, such alignment (dubbed later as the "disk of satellites", DoS) is prohibitively rare in computer simulations of galaxy formation in the Universe full of Dark Matter: the accreted subhalos should have had enough time to relax in the Milky Way's potential, thus erasing any signs of coherence.

It is, however, certainly too naive to believe that in $\Lambda \mathrm{CDM}$ Universe, the distribution of dwarf satellites around a Milky Way-like host is always isotropic. Zentner et al. (2005b) show that through the combined effect of i) filamentary accretion and ii) the alignment of sub-halo orbits with the major axis of the triaxial host halo, the probability of choosing the simulated subhalo populations from an isotropic distribution is as low as $10^{-4}$. The success of these simulations in assembling anisotropic satellite distributions is curious since these particular host galaxies do not posses disks. The presence of a baryonic disk should help to get rid of the satellites orbiting near it, thus making the satellite distribution more anisotropic. Libeskind et al. (2005) use a slightly different numerical setup to generate their host halos as well as their satellite galaxies but come to the same conclusion: a good fraction of the brightest satellites is bound to end up in a plane-like arrangement having arrived to the host through 1 or 2 primary filaments.

While Lynden-Bell (1976) only briefly mentions a possible scenario in which the parent galaxy dissolves to leave several smaller fragments behind

to be observed today as dwarf satellites, Kroupa et al. (2005) go further to 
suggest the exact mechanism responsible for their production. They speculate that the creation and the subsequent compression of the gaseous tidal tails is followed by tail fragmentation and active star-formation. It is claimed that the stellar systems born in this violent process, also known as tidal dwarf galaxies can survive long enough. If they do, their anisotropic distribution on the sky is merely the consequence of the proximity of their birthplaces in the tidal tail that is now vanished. This dSph formation mechanism advocated by Kroupa et al. (2005) harks back to their earlier dynamical work (Kroupa, 1997), in which a quasi-stable solution for a dSph-like DM-free stellar system is discovered. With the help of a suite of simple N-body simulations, it is argued that a tidal dwarf galaxy in the last throws of disruption can posses apparent surface brightness and velocity dispersion not unlike those observed in dSphs around the Milky Way. As Kroupa (1997) argues such high velocity dispersions would lead to over-estimated masses and therefore to highly inflated mass-to-light ratios, while the actual $M / L$ remains quite low. Metz and Kroupa (2007) re-run the experiment and show that their simulated tidal dwarf remnants and the Galactic dwarfs can look alike, especially within the region of the structural parameter space occupied by the ultra-faint satellites. Even though the fact of the existence of such out-ofequilibrium satellite configurations in numerical simulations is established, as of today, no evidence has been found that they can persevere for longer than a 1-2 Gyrs (see e.g. Casas et al., 2012).

As the census of the sub-structure in the halos of the Milky Way and the Andromeda galaxies is being filled in fast, the growing sample of satellites and streams allows for more rigorous tests of possible anistropies in their spatial and kinematic distributions. For example, Pawlowski et al. (2012) extend the study of the Galactic "disk of satellites" to include the known stellar and gaseous streams. Their argument in support of the previously found DoS orientation is that 7 out the 14 streams they analyse align well with the disk. With this observation in hand, they claim that it is not merely the "disk of satellites" that surrounds the Milky Way, but rather a "vast polar structure" (VPOS) appears to dominate the Galactic sub-system distribution at all radii. Once again the conclusion is reached that the presence of such structures is in contradiction with the $\Lambda C D M$ theory. Before the probability of encountering this so-called VPOS is worked out for the current galaxy formation paradigm, it is worth noting that while the number of the streams contributing to it seems large (a half of the total considered), their combined mass is minuscule. Therefore, these (in particular stellar) streams contribute 
close to nothing to the significance of the supposed anisotropy in the Galactic halo.

Curiously, in the case of the M31, Ibata et al. (2013) exhibit plausible evidence for the planar alignment of nearly half of the dwarf satellites. Moreover, these appear to be co-rotating around Andromeda in a semblance of a disk, which contains the line connecting the host galaxy and the Milky Way. This discovery is responsible for another attempt to debunk $\Lambda C D M$ this time by Hammer et al. (2013) who develop their earlier idea of a major merger at the M31 location (see e.g. Hammer et al., 2007) and suggest that most of the dwarf galaxies, including the Magellanic Clouds have formed as a result of this upheaval.

Overall, it seems that the hypothesis in which dwarf satellites are born in major merger events can give a convincing account of the observed distribution of satellites on the sky. However, currently the theory does not stack up against the entirety of the observational evidence, both locally (e.g. the extended star-formation histories and the extremely old stellar populations of the Milky Way dwarfs) as well as outside the Galaxy (e.g. low major merger rates for $\mathrm{L}_{*}$ hosts).

\section{Archaeologist's toolbox}

Low-mass stars (around $\sim 1 M_{\odot}$ ) shine for billions of years, and therefore keep the record of historical events in the Milky Way. To be able to read into the Galactic diary, collections of stars with comparable chemistry, age or, at least, similar luminosity class must be identified. The distributions of such stellar tracers in two (positions on the sky), three (place on the sky and along the line of sight), four (location in space and in radial velocity) or even seven (configuration space and velocity space coordinates together with chemistry) dimensions are then measured to benchmark, with some help from Galactic Dynamics, the theories of structure formation.

The Galaxy endlessly churns the pieces of smaller satellites it acquires, continuously smoothing the spatial densities of the debris. The rate at which the Galactic blender operates decreases from the centre outwards. Far out in the halo, where the orbital periods reach giga-years, unbound stellar substructures can maintain superficial spatial coherence for eons. However, closer to the Solar radius, extra (dynamical or chemical) information is required to filter out particular debris from the smooth mess. Therefore, the 

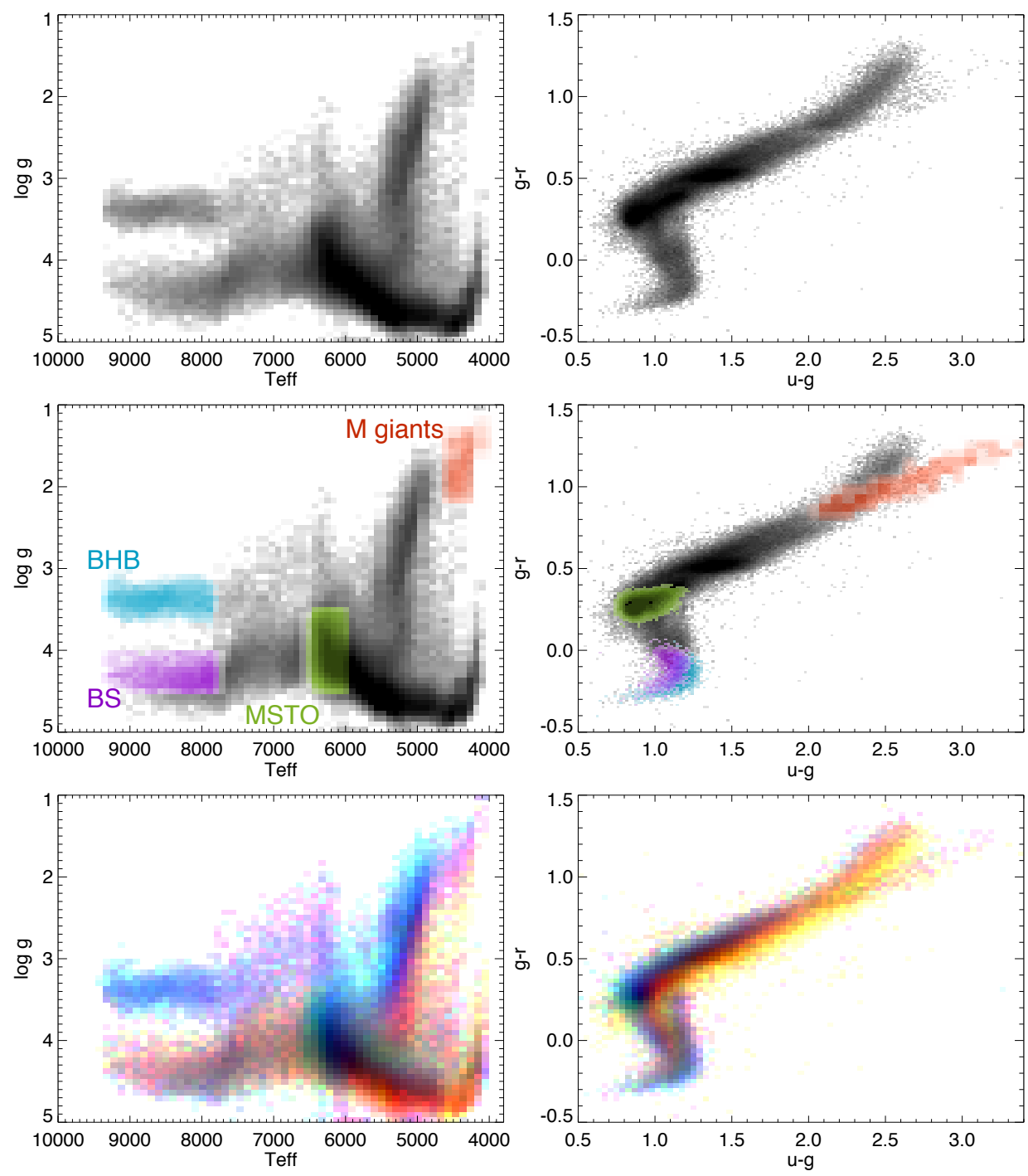

Figure 2: Stellar tracer selection in the SDSS database. Left: Density of stars in the plane of surface gravity $\log g$ and effective temperature $T_{\text {eff }}$ for $\sim 180,000$ DR 8 spectra with $15<g<17.5$. Right: Stars with spectroscopy from the left column are plotted on the plane of $u-g$ and $g-r$ color. Top: overview of the sample, darker shades of grey indicate higher density. Middle: Selecting the tracers. BHB (blue), Blue Straggler (violet), MSTO (green) and M-giant (red) stars are chosen in the left column based on their temperature and surface gravity. Density of selected stars is then over-plotted in $u-g, g-r$ space using the same color scheme. Bottom: Metallicity distribution in the sample. This shows false RGB images (left and right) constructed with 3 grey-scale density distributions of stars picked based on their $[\mathrm{Fe} / \mathrm{H}]$. Red component is for metalrich stars with $-0.75<[\mathrm{Fe} / \mathrm{H}]<0$, green (intermediate) $-1.5<[\mathrm{Fe} / \mathrm{H}]<-0.75$ and blue (metal-poor) $-3<[\mathrm{Fe} / \mathrm{H}]<-1.5$. 
interplay between the number of useful stellar tracers, the information content per star, and the overall volume probed is what determines the relevance of a Galactic halo survey.

In the not-so-distant future, with the data from the Gaia astrometric space mission and a host of planned large-area spectroscopic surveys, it should be possible to paint the unambiguous picture of the events that took place in the Galaxy between redshift $z=20$ and redshift $z=0$. At the moment, we will have to make do with what we have got. The observational advances in Galactic Archaeology made in the last few years happened thanks to a handful of wide area imaging surveys, namely 2MASS and SDSS, and massive spectroscopic efforts such as Segue and RAVE.

Of the several sky surveys of past decade, the SDSS appears to have been operating in a sweet spot: it turns out a 54 second exposure is long enough to reach Main Sequence stars at distances of several tens of kpc from the Sun, and thus yield an unprecedented 100 million object database; yet short enough to see plenty of the sky in limited amount of time. The now classic ugriz filter set encodes the stellar spectral energy distribution (SED) into a compact form, but preserves enough frequency diversity to study in detail a variety of stellar populations. This section therefore mostly concentrates on the observed properties of the Galactic stellar halo as seen by the SDSS (and its extensions) outside the Solar radius.

\subsection{Stellar tracers of the Galactic halo in the SDSS}

There are at least three species of stellar tracers available in the SDSS photometric data that a Galactic archaeologist can put to work. In order of decreasing population size, increasing luminosity and decreasing contamination, these are: Main Sequence Turn Off (MSTO) stars, Blue Horizontal Branch (BHB) stars and $\mathrm{M}$ giant stars. Figure 2 gives the whereabouts of each of these three in the space of stellar atmosphere parameters and the space of broad-band colors.

The left column of the Figure shows the logarithm of density of a sample of bright $(15<g<17.5)$ stars in the spectroscopic Data Release 8 of the SDSS (Aihara et al., 2011) on the plane of surface gravity $\log g$ and effective temperature $T_{\text {eff }}$. The density distribution of stars in this analog of the familiar Hertzsprung-Russell diagram is dominated by the pitch-black ribbon of the Main Sequence (MS), covering the range of $6500 \mathrm{~K}<T_{\text {eff }}<4500 \mathrm{~K}$. The sharp edge to the MS feature at high temperatures corresponds to the MS turn-off - these are the brightest of the MS stars and so, ideal for the halo 

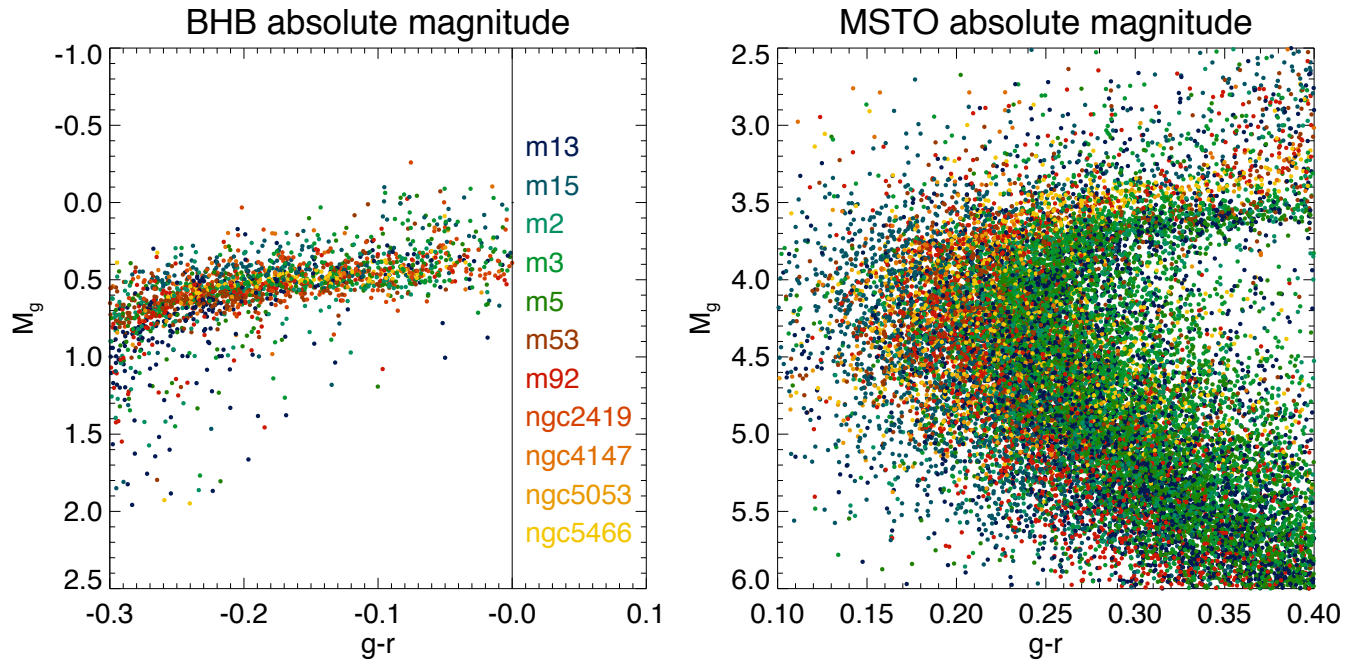

Figure 3: Absolute magnitude of stellar tracers. Left: Blue Horizontal Branch star candidates in 11 Galactic star clusters. Each dot represents one BHB, stars from different clusters are marked with different color. Cluster name and the color convention are shown in the inset. Once a model for the slight variation of the luminosity with color has been applied, the absolute magnitude of a BHB star can be estimated with accuracy $\lesssim 0.1 \mathrm{mag}$. Right Stars with $g-r<0.4$ in 11 Galactic star clusters. Apart from the variation by $\pm 0.5 \mathrm{mag}$ around the mean magnitude of the turn-off $M_{g} \sim 4$ due to age and metallicity differences between clusters, stars on the MS with lower luminosity as well as Sub-giant stars bright with higher luminosity are picked up by this $g-r$ cut. This results in the overall asymmetric spread of $\sim 3$ mag in $M_{g}$. 
exploration. The two faint and fuzzy clouds at temperatures above $7000 \mathrm{~K}$ are the helium burning Blue Horizontal Branch stars, with $3.7<\log g<3$, and the "reinvigorated" hydrogen burning pseudo-MS stars also known as Blue Stragglers, with $4<\log g<5$. Finally, the cool and inflated stars populate the Red Giant Branch, attached to the MS at around $T_{\text {eff }} \sim 6,500 \mathrm{~K}$ and reaching as high up as $\log g \sim 1.5$. There, right at the tip sits the small group of $\mathrm{M}$ giants, with $T_{\text {eff }}<5000 \mathrm{~K}$. To guide the eye, the stellar populations mentioned above are marked in color in the middle panel of the Figure.

As of DR8, only $\sim 0.2 \%$ of all detected stars have been targeted with SDSS spectroscopy. Therefore, to make surveying the Galaxy's halo practical, stellar tracers need to be identified by means of broad-band photometry only. To illustrate the photometric selection, the right column of Figure 2 gives the logarithm of the stellar density in the color-color space of $u-g$, $g-r$. Exactly the same bright stars, those with SDSS spectra, as used for the creation of the left column are plotted here. As expected, the behavior of the broad-band color distribution is to do with the locally measured slope of the SED, and hence is driven by the stars' temperature. Dwarf and giant stars are not easily separable anymore as they collapse to form one stellar locus, running from $g-r \sim 0.3$ to $g-r \sim 1.3$. However, it transpires that around the corners of the locus, the familiar populations can be picked up with ease.

The MSTO stars, being the hottest denizens of the MS, are clumped right at the blue edge of the $g-r$ distribution as evidenced by the tight green cluster in the right middle panel of the Figure. It is obvious that a simple $g-r<0.4$ cut would produce a relatively clean sample of the MSTO tracers. Still bluer in $g-r$, deviating downwards from the MSTO corner of the stellar locus, lies a "claw" of A-colored but old stars, BHBs and BSs. On further look, following their loci (marked in blue and violet) as they curve in $u-g, g-r$ space, some overlap between the two populations is visible, but more importantly, in $u-g$ color primarily, the BHB and the BS ridgelines stand separated by some 0.15 mag. Yanny et al. (2000) provide an efficient $u-g, g-r$ cut which yields a sample of BHB tracers with minimal contamination from BS or MSTO stars. Unlike the ubiquitous MSTO stars, the BHBs are manifestations of an old and metal-poor stellar population, as represented by their abundance in the Galactic globular clusters.

Even though telling a dwarf star from a giant star photometrically is pretty much impossible across a wide range of SDSS color, luminous and 
metal rich $\mathrm{M}$ giants stars peel away and redward from the stellar locus at around $u-g \sim 2.5$ as the red streak in the right middle panel of Figure 2 indicates. Equation 1 in Yanny et al. (2009) stipulates the M giant selection boundaries in the SDSS colors. Interestingly, age-wise M giants provide a probe of the halo complementary to that offered by old MSTO and BHB stars. As shown by Bellazzini et al. (2006) the stars ages range between 5 and 9.5 Gyr, with an average age of 8 Gyr.

\subsection{Chemical abundances of the SDSS stellar halo tracers}

What are the metallicity biases induced by the particular choice of the stellar tracers described above? The bottom row of the Figure 2 shows the metallicity $[\mathrm{Fe} / \mathrm{H}]$ distribution of the bright SDSS DR8 stars with spectra. Stars are split in three groups according to their $[\mathrm{Fe} / \mathrm{H}]$ and greyscale density distributions in $\log g, T_{\text {eff }}$ and $u-g, g-r$ are produced. Then the three greyscale pictures are combined to produce two false-color images, one for the left column and one for the right. The red components in the false RGB images are based on metal-rich stars with $-0.75<[\mathrm{Fe} / \mathrm{H}]<0$, for the green components intermediate metallicity stars are selected with $-1.5<$ $[\mathrm{Fe} / \mathrm{H}]<0.75$, finally the most metal-poor stars with $-3<[\mathrm{Fe} / \mathrm{H}]-1.5$ contribute to the blue components of the images. Therefore, clumps of stars that are mostly blue in the bottom row of the Figure are mostly metal-poor, the red features are made up of mostly metal-rich stars, with other colors corresponding to $[\mathrm{Fe} / \mathrm{H}]$ mixtures in between. In particular, stars in all three metallicity bins contributed roughly equal amounts to pixels with dark grey or almost black color.

The lower left panel of Figure 2 confirms that, as expected, the BHBs are predominantly metal-poor, the $\mathrm{M}$ giants are metal-rich and the MSTO have no particular metallicity bias. The right panel in this row showcases beautifully the discriminating power of the SDSS broad-band filters: the pixels on the stellar locus can be seen to change their color dramatically depending on their $u-g, g-r$ values. This means that a unique $[\mathrm{Fe} / \mathrm{H}]$ value can be assigned to a MS star given its ugr measurements. The idea of photometrically derived metallicities is the same idea that is behind the UV-excess method first applied to interpret the chemo-dynamical properties of the Galactic halo stars by Eggen et al. (1962). Ivezić et al. (2008) develop polynomial models (updated recently by Bond et al. (2010)) to calculate photometric metallicities from SDSS ugr measurements for F and G Main Sequence stars in the range of $0.2<g-r<0.6$. 


\subsection{Absolute magnitudes of stellar tracers}

One of the primary advantages of mapping the galaxy in which we actually reside is the access to the third spatial dimension. While most other galaxies appear to us in a cartoonish 2D, distances to the Milky Way stars can be measured using the annual parallax or with much cheaper (but less accurate) photometric parallax (see e.g. Jurić et al., 2008).

Figure 3 reveals exactly how much uncertainty there exists in determining the luminosities of BHB and MSTO stars using their broad-band colors only. The left panel of the Figure shows variation in $g$-band absolute magnitude $M_{g}$ as a function of $g-r$ color for BHB candidate stars in 11 Galactic star clusters imaged in the SDSS ugriz filters (An et al., 2008). Regardless of the (small) metallicity differences and irrespective of the (modest) age spread, the BHBs form a tight sequence with a gentle slope in $g-r$. The changes in $M_{g}$ from slightly above $M_{g}=0.5$ at red colors to slightly below $M_{g}$ at blue colors can be approximated with a simple polynomial model to give the BHB absolute magnitude within $0.1 \mathrm{mag}$ (see e.g. Deason et al., 2011).

The simple $g-r<0.4$ cut picks up a whole slew of stars of various luminosities as evidenced by the right panel of Figure 3. These range from bright sub-giants at $M_{g} \sim 3.5$, through the actual MSTO stars with $3.5<M_{g}<4.5$, to dwarfs on the Main Sequence that are as faint as $M_{g} \sim 6$. Additionally, even though the star clusters in the sample considered do not cover the whole range of metallicity or age, matching quite well the old and metal-poor Galactic halo, the $[\mathrm{Fe} / \mathrm{H}]$ and age differences result in significant shifts in both $g-r$ and $M_{g}$ around the MS turn-off. As a result, the overall absolute magnitude spread for the tracers selected is of the order of 3 magnitudes.

It is obvious from the right panel of Figure 3 that for a star on the Main Sequence, an estimate of the absolute magnitude can be obtained from the value of its $g-r$ color. The dimming of dwarf stars with lowering temperature is the basis for the so-called photometric parallax method, which actually does not have anything in common with the annual parallactic motion, apart from the fact that it also delivers the stellar distance. Jurić et al. (2008) takes advantage of the superb quality of the SDSS photometry and calibrates the absolute magnitude of MS stars using the color-magnitude behavior of stars in the Galactic globular clusters (GC) with well-determined distances. Such distance estimate can be further improved, as shown by (Ivezić et al., 2008), if the photometrically-derived metallicity is included. However, as shown by Smith et al. (2009, 2012), around the MSTO the absolute magnitude calibration provided by Ivezić et al. (2008) suffers from considerable bias. To 
remedy this, Smith et al. (2009, 2012) offer an appropriately flexible method to tune the absolute magnitudes of stars around the turn-off according to their metallicity.

Finally, as regards to $\mathrm{M}$ giants, these stars are too luminous, too metalrich and too young to be found in the Milky Way's globular clusters, and hence, the methods of absolute magnitude calibration discussed above do not apply. However, Yanny et al. (2009) show that using the pieces of the Sagittarius stream based on the distances measured with RR Lyrae one can calibrate the $\mathrm{M}$ gaint absolute magnitude to obtain $M_{g} \sim-1$. Note, however, that this is only valid for a particular mix of metallicity and age similar to that of the Sagittarius debris.

\subsection{Matched Filter approach}

Rather than selecting stars in a particular luminosity class and/or metallicity range to trace the stellar halo sub-structure, an alternative popular approach is to use the entirety of the stellar populations belonging to the satellite that is assumed to be disrupting or disrupted. The probability of any star in the halo to come from the desired population is obtained by simply taking the ratio of stellar density in bins of color and magnitude of the satellite and of the background. These probabilities (or weights) are then binned on the sky and the smooth slowly-varying component of the density contributed by the background is subtracted. This so-called Matched Filter technique as pioneered by Grillmair et al. (1995) has been employed with great success to isolate extra-tidal congregations of stars around many Milky Way companions (e.g. Odenkirchen et al., 2001; Rockosi et al., 2002; Niederste-Ostholt et al., 2010a; Sollima et al., 2011). The method can deliver superb results, but has two inherent breaking points: i) for satellites that are completely dissolved in the Galactic gravitational potential, no template color-magnitude density is available, and ii) as the stars from a disrupting object normally cover a large area on the sky their heliocentric distances change and therefore the probabilities assigned by the method will not match those in the debris everywhere. While the first problem can be easily overcome by searching for the best-matching CMD template by trial and error as demonstrated beautifully by Grillmair and Dionatos (2006b), there is no simple (and elegant) remedy to the issue of evolving distance. 


\section{Stellar halo of the Galaxy}

\subsection{Evidence of sub-structure in the stellar halo}

\subsubsection{The Field of Streams}

The broad-brush spatial properties of the sub-structure in the Milky Way's halo as seen by the SDSS in its DR9 incarnation (Ahn et al., 2012) are displayed in Figures 4 and 5 in Equatorial and Galactic coordinates respectively. The SDSS DR5 version of this false-color halo map dubbed the Field of Streams is published by Belokurov et al. (2006b). Included in this map are predominantly MS and MSTO stars, which are selected with a simple color cut $g-i<0.6$ (a selection almost identical to the described above $g-r<0.4$ ). These stars are then split into three equal magnitude bins between $i=19$ and $i=22.5$. Three grey-scale images each showing stellar tracer density in a particular magnitude bin (see bottom row of the Figures) are then combined in the order that maps bright-intermediate-faint stars onto blue-green-red channels. Given the fixed $g-i$ cut, the false RGB color of each pixel can be interpreted as a rough estimate of the average heliocentric distance of the stars contributing to it. Assuming, very approximately, that the typical $M_{i} \sim 4$. for the selected tracers, given the magnitude range of $19<i<22.5$, the range of the heliocentric distances probed is $10<D(\mathrm{kpc})<50$.

Most of the contiguous sky coverage in the SDSS footprint falls around the North Galactic Cap. The arc dominating the density map at the high Galactic latitudes in the North is the leading tidal tail emanating from the Sagittarius dwarf galaxy. The Sgr debris is also the only prominent structure in the stellar halo in the Galactic South, where the trailing tail of the dwarf can be seen. In Figure 4, the leading stream changes color from red to green-blue as its heliocentric distance drops from $\sim 50 \mathrm{kpc}$ at Dec $=220^{\circ}$

(just behind the Galactic centre) to $\sim 15 \mathrm{kpc}$ at Dec $=130^{\circ}$ (Belokurov et al., 2006b). There, at the Galactic anti-centre, the Sgr stream crosses the prominent Galactic Anti-Center Stellar Structure seen in the Figure as a violet-blue tilted band with striation. This complex configuration is due to the multiple components of the GASS: the so-called Monoceros Ring (e.g. Newberg et al., 2002), the Anti-Center Stream (e.g. Grillmair, 2006b; Grillmair et al., 2008) and the Eastern Banded Structure (Grillmair, 2006b). The fuzzy green haze directly underneath the Sgr stream at around RA 180 is the large cloud of stars dubbed Virgo overdensity (e.g. Jurić et al., 2008). The central, dense regions of the stellar halo can be seen as bright white-blue glow on either size of the Galactic disk at $320^{\circ}<\mathrm{RA}<220^{\circ}$. As Belokurov 

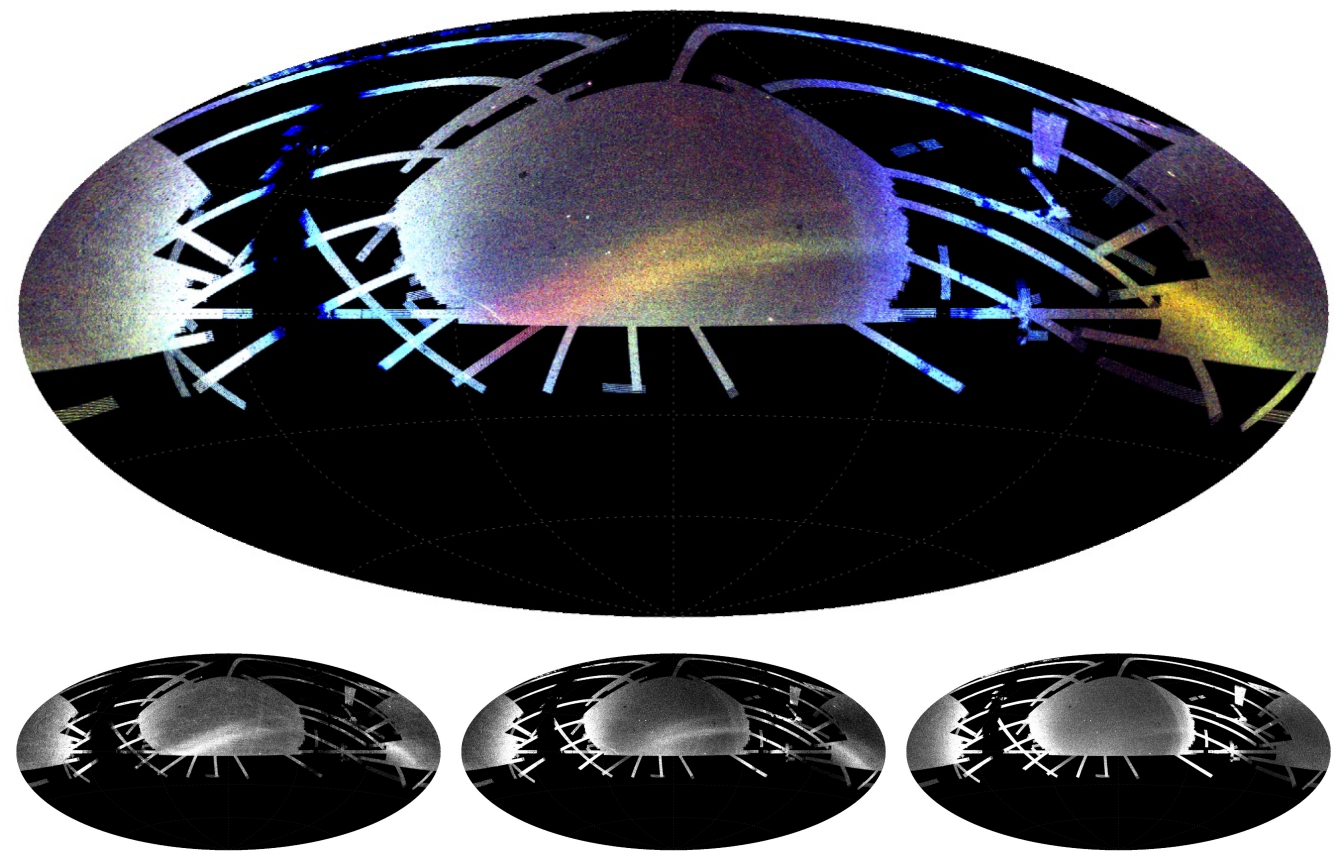

Figure 4: Map of the stellar halo of the Milky Way in Equatorial coordinates (centered on $\mathrm{RA}=180^{\circ}$ ) using the data from SDSS DR9. Top: False-color composite image uses $16,000,000$ predominantly MS and MSTO stars selected with a simple color cut $g-i<0.6$ and split into three equal magnitude bins between $i=19$ and $i=22.5$. Given the $g-i$ cut, the color of the pixel can be interpreted as a rough estimate of the average heliocentric distance of the stars contributing to it. White corresponds to high stellar densities in all three magnitude bins. Black corresponds to areas with missing data.Bottom: Grey-scale images each showing the stellar tracer density in a particular magnitude bin are then combined in the order that maps the densities of bright, intermediate and faint stars onto blue, green and red channels as shown in the Top panel. 

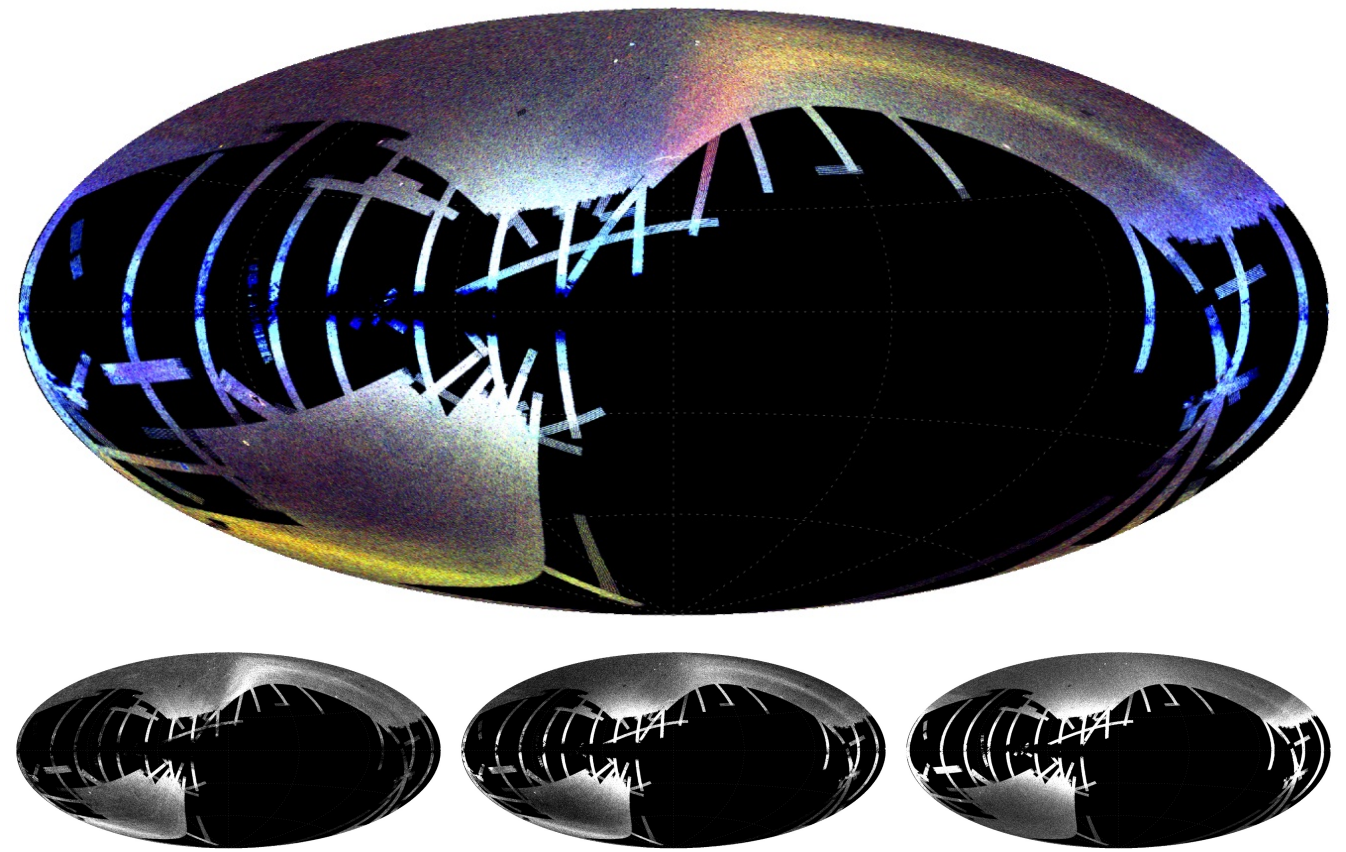

Figure 5: Same as Figure 5 but in Galactic coordinates. Galactic $l=0^{\circ}, b=0^{\circ}$ is at the centre of the Figure.

et al. (2007b) point out, the distribution of the MS and the MSTO stars does not peak in the direction of the Galactic centre as it seems offset towards the positive Galactic $l$. Moreover, on closer examination there appears to be a substantial asymmetry in the counts of MS/MSTO stars with heliocentric distances $10<D(\mathrm{kpc})<20$ between the Galactic North and the South. These observations lead the authors to the conclusion that a sizable portion of the central Milky Way halo could be due to yet another massive stellar sub-structure, so-called Hercules-Aquila Cloud.

A Galactic projection of the same map (see Figure 5) helps to make sense of some of the stellar halo density patterns. For example, it shows that the Sagittarius leading tail nearly misses the Galactic North, the GASS is mostly confined to Galactic latitude $b<30^{\circ}$. Additionally, even though the SDSS coverage at positive and negative Galactic longitude is not equal, it is clear that at $l>0^{\circ}$ at high $b>30^{\circ}$ there does not exist a counter-part to the Virgo overdensity. 


\subsubsection{The big 4}

The Sagittarius Stream, the Galactic Anti-center Stellar Structure, the Virgo and the Hercules-Aquila Clouds are the four largest stellar structures in the halo of the Milky Way. Out of these four, only the Sgr Stream lies predominantly outside the Galactic disk making it possible to estimate its total extent and the overall stellar mass. The Stream consists of two tails, the leading and the trailing, flowing from the Sgr dwarf galaxy, which currently lies on the opposite side of the Galaxy, behind the bulge, several degrees under the disk. The dwarf is falling onto the disk and has just passed its point of the nearest approach at $\sim 15 \mathrm{kpc}$ from the Galactic center. The two tails appear bifurcated (see e.g. Belokurov et al., 2006b; Koposov et al., 2012 ) and extend each at least as far as $\sim 180^{\circ}$ away from the progenitor (see Figures 4 and 5). The leading tail is traced as far as $50 \mathrm{kpc}$ from the Galactic center, while the apo-galacticon of the trailing debris is probably as far as 60-100 kpc. Both the Sgr remnant and the stream host a range of stellar populations with different ages and metallicities. In particular, along the stream, a substantial population gradient is observed (e.g. Chou et al., 2007; Yanny et al., 2009; Bell et al., 2010; Chou et al., 2010; Keller et al. 2010, Carlin et al., 2012), which, within any sensible model of the dwarf disruption, would mean a similarly pronounced abundance and age gradient in the progenitor. Using a variety of stellar tracers across the sky, Niederste-Ostholt et al. (2010b) map the Sgr debris and, correcting for the distance and the abundance gradients estimate the total stellar luminosity of the progenitor prior to disruption. They find that, before merging with the Galaxy, the dwarf was as bright as $1.5 \times 10^{8} M_{\odot}$ or just under $M_{V} \sim-16$, but today it has lost as much as $70 \%$ of its stars to the Galactic tides.

The Virgo Cloud can be seen as green haze directly underneath the Sgr Stream at around $R A \sim 12^{h}$. While early glimpses of this structure are reported in several studies, based on the SDSS DR4 imaging data, Jurić et al. (2008) provide the first large scale map of the Cloud and emphasize its gigantic extent on the sky of least $\sim 1000 \mathrm{deg}^{2}$. From the inspection of Figure 4, it is obvious that the portion of the Virgo Cloud analyzed by Jurić et al. (2008) is only the tip of the structure that appears to continue to lower Declinations as far as the SDSS/Segue imaging stripes can go. Accordingly, Bonaca et al. (2012b) take advantage of the extra imaging in the SDSS DR8 and claim that the true extent of the Cloud is somewhere between $2000 \mathrm{deg}^{2}$ and $3000 \mathrm{deg}^{2}$. The debris cover an enormous portion of the sky, but given 
the typical distance and the low surface brightness, the total luminosity of the Virgo Cloud is estimated to be modest $<10^{6} M_{\odot}$ (Bonaca et al., 2012b).

The Galactic Anti-Center Stellar Structure and the Hercules-Aquila Cloud have most of their stars at low Galactic latitudes: within $|b|<40^{\circ}$, GASS can be found at roughly $120^{\circ}<l<240^{\circ}$ and HAC at $20^{\circ}<l<70^{\circ}$ (see Figure 5. In fact, both of these structures appear to be stuck right in the plane of the disk as their candidate member stars are detected in both Northern and Southern hemispheres. Given such an awkward location in the Galaxy, it is still questioned whether all, or at least some of the signal attributed to these two can be explained away invoking variants of the known components of Milky Way. For example, it is claimed that parts of the GASS can well be ascribed to the Galactic flare and/or the warp (e.g. Ibata et al., 2003), and the HAC is really nothing but the asymmetric thick disk (e.g. Larsen et al., 2008, 2011). However, there exists additional observational data within which stellar over-densities are clearly seen in the directions of both the GASS and the HAC in tracers unlikely to populate either of the disks. For example, the distant portion of the GASS, the And-Tri stream is traced with $\mathrm{M}$ giants at distances of the order of $30 \mathrm{kpc}$. HAC can be picked up with RR Lyrae in the SDSS Stripe 82 dataset (e.g. Watkins et al., 2009; Sesar et al. 2010a) at $10<D<20 \mathrm{kpc}$. As most of the light in both GASS and $\mathrm{HAC}$ is hidden in the Galactic plane, only very approximate estimates of their total stellar masses exist in the literature. Belokurov et al. (2007a) give a conservative estimate of $\sim 10^{7} L_{\odot}$ for the Hercules-Aquila Cloud. For the closer portion of the GASS, Yanny et al. (2003) get the total stellar mass in the range of $0.2-5 \times 10^{8} M_{\odot}$, with the larger value obtained assuming that i) the GASS follows an exponential profile as a function of $\mathrm{z}$ and ii) encompasses the entire Milky Way. Several follow-up studies present the updated measurements of the structure and the stellar populations of the pieces of GASS visible in the SDSS (e.g. de Jong et al., 2010; Grillmair, 2011; Li et al., 2012) and in the deeper imaging (e.g. Conn et al., 2012). According to the body of work published so far, the components of the GASS most consistent with the accretion scenario (see e.g. Peñarrubia et al., 2005) have, overall, much flatter density distribution as a function of Galactic $|b|$ or $|z|$. If true, this observation would lead to the substantial reduction of the overall luminosity of the GASS. Perhaps, the following simple argument can be constructed to provide a complementary guess as to the total stellar mass in the GASS. Given that the parts of the GASS detected within the SDSS field of view typically have similar or lower surface brightness as compared to the 


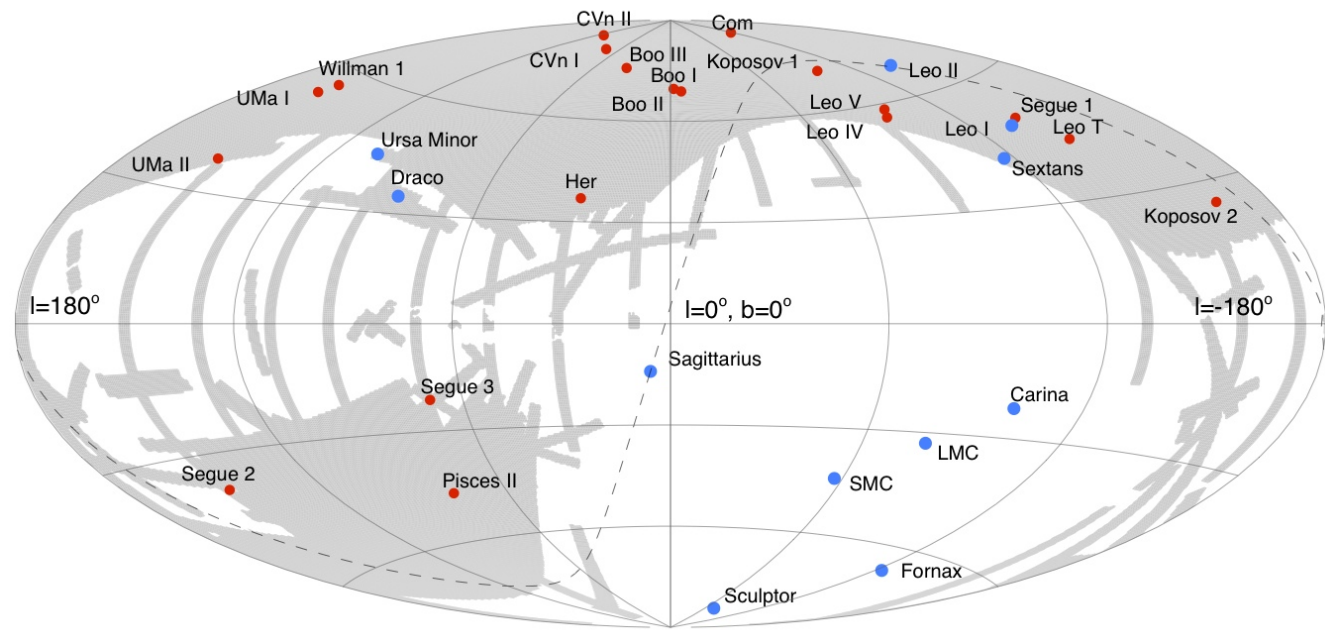

Figure 6: Distribution of the classical dwarf galaxies (blue filled circles) and the SDSS ultra-faint satellites (red filled circles), including three ultra-faint star clusters, in Galactic coordinates. The SDSS DR8 imaging footprint is shown in grey. Dashed line marks the tentative orbit of the Sgr dwarf galaxy. Galactic $l=0^{\circ}, b=0^{\circ}$ is at the centre of the Figure.

Sgr Stream, but are on average closer by a factor of $2-5$, it is not unlikely that the structure, in fact, contains more than $10^{8} M_{\odot}$.

\subsubsection{Ultra-faint satellites}

Visible as bright dots of different colors in the maps in Figures 4 and 5 are the compact stellar over-densities corresponding to the Galactic satellites that give the impression of being still intact. The brightest of these "hot pixels" correspond to the well-known star clusters and classical dwarf galaxies, while the very faint and barely visible small-scale over-densities mark the locations of the so-called ultra-faint satellites of the Milky Way. Although several of these, including Boo I, Boo III, CVn I and UMa II, are seen in this picture with a naked eye, the rest of the population of these objects is too insignificant and can only be unearthed via an automated over-density search. The first example of such an automated stellar over-density detection procedure is presented in Irwin (1994) who apply the method to the data from the photographic plates of the POSS I/II and UKST surveys scanned at the APM facility in Cambridge. A vast area of 20,000 square degree of the sky is searched but only one new nearby dwarf galaxy is detected, namely 
the Sextans dSph. A variant of the procedure is used, albeit with a little less luck, by Kleyna et al. (1997), and subsequently by Willman et al. (2005a b) who actually find the two very first examples of ultra-faint objects in the SDSS data. The ease with which these systems reveal themselves in a stellar halo density map akin to the "Field of Streams" (see Zucker et al., 2006a; Belokurov et al., 2006c) helped to re-animate the search for new Milky Way satellites and more than a dozen of new discoveries have been reported in quick succession (Zucker et al., 2006b; Belokurov et al., 2007c; Irwin et al., 2007; Koposov et al., 2007; Walsh et al., 2007; Belokurov et al., 2008, 2009; Grillmair, 2009; Belokurov et al., 2010). Figure 6 maps the distribution of all presently known SDSS ultra-faint satellites on the Galactic sky.

The accuracy and the stability of the SDSS photometry makes it possible for the over-density detection algorithms to reach exceptionally faint levels of surface brightness across gigantic areas of the sky. However, even though genuine Galactic satellites can be identified in the SDSS as groups of only few tens of stars, their structural parameters can not be established with adequate accuracy using the same data. Deep follow-up imaging on telescopes like INT, CFHT, LBT, Magellan, MMT, Subaru and most recently HST, has played a vital role in confirming the nature of the tiny stellar blobs in the SDSS, as well as in pinning down their precise sizes, ellipticities and their stellar content. The most recent, deep and wide photometric studies of a significant fraction of the new SDSS satellites are published by Okamoto et al. (2012) and Sand et al. (2012). They point out that even at distances $D>100$ kpc from the Galactic centre, the outer density contours of CVn II, Leo IV and Leo $\mathrm{V}$ display extensions and perturbations that are probably due to the influence of the Milky Way tides. Similarly, there is now little doubt that both UMa II and Her are excessively stretched, as their high ellipticities as first glimpsed at discovery (Zucker et al., 2006a; Belokurov et al., 2007c) are confirmed with deeper data (Muñoz et al., 2010; Sand et al., 2009). Note, however that apart from these two obvious outliers there does not seem to be any significant difference in the ellipticity distributions of the UFDs and the Classical dwarfs contrary to the early claims of Martin et al. (2008). This is convincingly demonstrated by Sand et al. (2012) with the help of the imaging data at least 2 magnitudes deeper than the original SDSS. They, however, detect a more subtle sign of the tidal harassment: the preference of the density contours of the SDSS satellites to align with the direction to the Galactic centre.

As far as the current data is concerned, the SDSS dwarfs do not appear 
to form a distinct class of their own, but rather are the extension of the population of the Classical dwarfs to extremely faint absolute magnitudes. However, as more and more meager luminosities are reached, it becomes clear how extreme the faintest of the UFDs are. The brightest of the group, CVn $\mathrm{I}$ and Leo $\mathrm{T}$ show the usual for their Classical counter-parts signs of the prolonged star-formation. For example, CVn I hosts both Blue Horizontal Branch and Red Horizontal Branch populations, while Leo T shows off a sprinkle of Blue Loop stars. However, the rest of the ensemble appears to have narrow CMD sequences with no measurable color spread around the conventional diagnostic features, e.g. MSTO and/or RGB, thus providing zero evidence for stellar populations born at different epochs (e.g. Okamoto et al. 2012). The CMDs of the UFDs have revealed no secrets even under the piercing gaze of the HST: all three objects studied by Brown et al. (2012) appear to be as old as the ancient Galactic globular cluster M92. Yet the low/medium and high-resolution follow-up spectroscopy reveals a rich variety of chemical abundances somewhat unexpected for such a no-frills CMD structure. The first low-resolution studies of Simon and Geha (2007) and Kirby et al. (2008) already evince the existence of appreciable $[\mathrm{Fe} / \mathrm{H}]$ spreads in the SDSS dwarfs with the metallicity distribution stretching to extremely low values. Analyzing the medium and high resolution spectra of the Boo I system, Norris et al. (2010) measure the spread in $[\mathrm{Fe} / \mathrm{H}]$ of $\sim 1.7$ and the $[\mathrm{Fe} / \mathrm{H}]$ dispersion of $\sim 0.4$ around the mean value of -2.55 at $M_{V} \sim-6$. It seems that this behavior of decreasing mean metallicity with luminosity while maintaining a significant enrichment spread is representative of the UFD sample as a whole (see also Lai et al., 2011; Koch et al., 2013; Vargas et al., 2013). Crucially, these spectroscopic observations require that, notwithstanding their low stellar luminosities at the present day, these satellites had enough total mass in the past to hold on to some of the enriched gas after the first supernovae explosions and subsequently produce more stars. Additionally, in the UFDs, the downwards shift of the mean metallicity with decreasing stellar mass reveals that they can not simply be direct analogs of the Classical dwarfs stripped off the bulk of their stellar content.

Of the 16 ultra-faint satellites currently known, only 5 systems have a handful of stars studied with high-resolution spectroscopy. More specifically, one star in Leo IV (Simon et al. 2010), two stars in Her (Koch et al., 2008), 3 stars in each of UMa II and Com (Frebel et al., 2010) and 7 in Boo I (Gilmore et al., 2013) have been measured so far. It is perhaps too early to draw far-reaching conclusions from these highly incomplete data, nonethe- 
less an interesting picture seems to be emerging from the detailed abundance work. Although wanting in quantity, these high-resolution high-quality spectroscopic data do robustly confirm the key properties of the UFD chemical enrichment histories hinted at by the analysis of the low-resolution (and at times, low-S/N) samples. The SDSS dwarfs are indeed characterized by remarkably low levels of the overall iron enhancement as well as the heterogeneity of the individual stellar abundances (in each of the 4 satellites that have more than 1 star measured). Additionally, the very first high-resolution study of a UFD by Koch et al. (2008) reported a depletion of heavy neutron capture elements. RGB stars with low abundance levels of barium are also found in Leo IV, Com, UMa II and Boo I (Simon et al., 2010; Frebel et al., 2010; Gilmore et al., 2013). Moreover, in Boo I, several extremely metal-poor stars are demonstrated to have increased levels of carbon (see e.g. Norris et al., 2010). Potentially, there are at least two notable implications of these enrichment patters. First, carbon-enhanced metal-poor stars are common denizens of the Galactic stellar halo, yet if there occur any in the classical dSphs, they have so far eluded the detection. The existence of such stars in both the UFDs and the MW stellar halo may signify the commonality of the chemical evolution paths of the halo progenitor(s) and the ultra-faint satellites. Second, as several authors have pointed out (e.g. Koch et al., 2008; Simon et al., 2010, Frebel et al., 2010), the enhancement in $\alpha$-elements together with the depletion in neutron-capture elements at low metallicities can be linked to the products of the Population III SNe, therefore implying that a good fraction of the stellar content in the UFDs could be direct descendants of the first stars (see also Frebel and Bromm, 2012).

It is evidently not possible to come up with a sensible theory of the UFD formation without an idea of their total masses. Such a measurement, which necessarily involves accurate kinematics for a large enough sample of the satellite members, is, however, not straightforward. This is simply due to the fact that, as illustrated by Koposov et al. (2008), the majority of these objects are discovered very close to the detection boundary, implying that the over-density signal is dominated by the stars close to the SDSS detection limit of $r \sim 22$. At these magnitudes, only half a handful of facilities in the world are capable of obtaining absorption spectra of signalto-noise sufficient to measure the line-of-sight velocities of individual stars. Even if the kinematic signal is present in the data, winnowing it out from the low-resolution spectra of low-metallicity stars is a challenge. An even harder challenge is figuring out the uncertainties of the velocity measurements. For 
most ultra-faints, the typical member velocity uncertainty is of the order of, or larger than, the intrinsic velocity dispersion of the system. Under or overestimating the measurement error by a small fraction can lead to a substantial systematic velocity dispersion bias, and as a consequence, a wrong aperture mass. Despite the above mentioned difficulties of the task at hand, several teams report the results of their heroic attempts to gauge the central masses of the UFDs (e.g. Martin et al., 2007a; Simon and Geha, 2007; Walker et al., 2009; Belokurov et al., 2009; Simon et al., 2011; Koposov et al., 2011)

The structural parameters of the faintest of the SDSS satellites, e.g. Willman 1, Segue 1 and 2, Boo II are dangerously similar to those of the most diffuse star clusters in the Milky Way and M31. It is not conceivable, purely on the basis of their size or luminosity, to come up with the most likely scenario of their formation. Therefore, their kinematic and chemical properties are the most important clue. Today, for the faintest objects, it is just possible, after many hours spent on Keck and VLT, to build datasets with radial velocities for a dozen or two of the MSTO members and a trickle of the Red Giants. Accordingly, the most recent and the most thorough kinematic analysis of Willman 1, Segue 1 and Segue 2 can be found in Willman et al. (2011); Simon et al. (2011) and Kirby et al. (2013) correspondingly. Moreover, Norris et al. (2010) independently carries out a thorough chemical study of Segue 1 using a different combination of the telescope, the instrument and the analysis techniques. For these three best studies objects, the picture does not appear to be as clear-cut as for their more luminous peers. For example, the evolution of the line-of-sight velocity with radius in Willman 1, where the inner-most stars are offset by some $8 \mathrm{~km} / \mathrm{s}$ from the outer-most ones is unusual, and is, perhaps, a sign of the advanced stage of tidal disruption. There is also an evidence of the spread in $[\mathrm{Fe} / \mathrm{H}]$, but unfortunately it is based on the measurements of only two Red Giant Branch stars.

Segue 1, the best studied of the three, has a substantial velocity dispersion at $3.7_{-1.1}^{+1.4} \mathrm{~km} / \mathrm{s}$ and an impressive metallicity spread. There are however some quirks with regards to both the velocity and the metallicity dispersion measurements, such as the fact that the velocity dispersion calculated using the brightest members only ( 5 red giants stars) is essentially consistent with zero, or the fact that some of the most metal-poor stars also lie several halflight radii away from Segue 1's center (see Norris et al., 2010). Perhaps more significant is the observation by Newberg et al. (2010) that the Orphan stellar stream runs at the identical distance and velocity only $\sim 2$ degrees away from the position of Segue 1. Given the width of the stream of 1 degree, a 
significant contamination of spectroscopic samples at Segue 1's location is not very likely. Yet, the dynamical association between the two is, however, quite possible: both the progenitor of the Orphan Stream and Segue 1 itself might have been parts of a bigger system which is now completely disrupted.

The evidence of such an accretion event is even more dramatic in the case of Segue 2. Taking into account the observations reported in Majewski et al. (2004); Rocha-Pinto et al. (2004), Segue 2 is immersed in the debris of the Triangulum-Andromeda stream, which is interpreted as the distant (at $\sim 30$ $\mathrm{kpc}$ compared to $34 \mathrm{kpc}$ for Segue 2) counter-part of the Monoceros stream and part of the larger Galactic Anti-Center Stellar Structure. As published by Rocha-Pinto et al. (2004), the velocities of $\mathrm{M}$ giant members of Tri-And structure are $0<V_{G S R}<60$ in the range of longitudes $160^{\circ}<l<130^{\circ}$ at the Galactic latitudes slightly lower than that of Segue 2. This velocity distribution can be modeled as a Gaussian that peaks around $V_{G S R} \sim 30 \mathrm{~km} \mathrm{~s}^{-1}$ which is a good match to the measurement of the satellites line-of-sight velocity $V_{G S R} \sim 40 \mathrm{~km} \mathrm{~s}^{-1}$. The coverage of the area with the spectroscopic M giants is sparse, and the SDSS spectroscopic footprint is seriously incomplete here. However, Belokurov et al. (2009) present an unambiguous kinematic evidence for the stream's existence using the spectra obtained with 1 degree wide field Hectochelle instrument on MMT. They claim that the stream's stars are more metal-rich on average and their velocity distribution can be described with a broader Gaussian, namely $15 \mathrm{~km} / \mathrm{s}$ vs $\sim 3 \mathrm{~km} / \mathrm{s}$ for Segue 2 . Most recently, Kirby et al. (2013) re-evaluated the spectroscopic properties of Segue 2 albeit with a different observational setup and a smaller field of view as compared to the original study of Belokurov et al. (2010). They claim no detection of the stream signal, which is perhaps not surprising given the targeting strategy and the area of the sky surveyed. Intriguingly, they measure much lower velocity dispersion (essentially consistent with zero), thus markedly reducing the central mass of the satellite.

\subsubsection{Star cluster streams}

The large undissolved stellar clouds (Virgo, Hercules-Aquila) and broad long streams (Monoceros, Sagittarius) described earlier are the primary contributors to the Galactic halo in terms of the stellar mass. In the past decade, an assortment of much narrower, often shorter and significantly less luminous streams has been identified. It seems most likely that these would have originated in star clusters. Some of these wispy tidal tails are discernible in Figures 4 and 5, such as the tidal tails of the Palomar 5 globular cluster 
(Odenkirchen et al., 2001; Grillmair and Dionatos, 2006a). However, in their majority these feathery streams require a more subtle approach and are best seen with the help of the Matched Filter technique. Some of the star cluster debris have obvious progenitors like the short stubby tails visible around e.g. NGC 5466 (Belokurov et al., 2006a), NGC 5053 Lauchner et al. (2006), Pal 14 (Sollima et al., 2011), Pal 1 (Niederste-Ostholt et al., 2010a). For the others, typically extending many degrees on the sky, no suitable progenitor has been discovered yet, e.g. the GD-1 stream (Grillmair and Dionatos, 2006b), a group of four streams Styx, Acheron, Cocytos, Lethe (Grillmair, 2009) and the most recently identified Pisces Stellar Stream (Bonaca et al. 2012a; Martin et al., 2013).

It is interesting to estimate the total number of star clusters that have disrupted so far and whose stars are now part of the Galactic halo. While such a count is valuable as it gives an idea of the fraction of the halo that is comprised of the GC debris, it is not straightforward as it requires the knowledge of the Cluster Initial Mass Function (CIMF) and a model of the cluster evolution in the Milky Way tidal field. An example of such calculation is presented in Alexander and Gieles (2013) who approximate the CIMF with a power-law distribution and apply the semi-analytic model of Gieles et al. (2011) for the star cluster evolution in a logarithmic Galactic potential. They find that, of the several models they consider, the Roche volume under-filling model with a flat CIMF (power law index 0) reproduces the present day properties of the Milky Way's GCs the best. While the authors do not give the exact number of dissolved clusters, it is clear that the flat mass function evolution can only produce a moderate number of star cluster streams in the Galactic halo, perhaps orders of magnitude less as compared to the rising power laws (e.g. -2). Alternatively, the number of the GC streams detected so far with the SDSS could be translated into a Galaxy total if there existed an estimate of the stream detection efficiency. However, it is possible that a significant fraction of the known long and narrow stellar streams may have been produced as a result of only a few accretion events. For example, given the noticeable alignment of their orbital planes, it is feasible that the progenitors of the Styx, Acheron, Cocytos and Lethe streams arrived to the Galaxy together with a much bigger satellite. The fact that the GC accretion is most likely linked to the infall of more massive Galactic satellites is another reason to believe that the total number of GC streams is relatively low given the evidence for the uneventful Milky Way's mass assembly history. 


\subsubsection{Orphan and Styx. Streams from ultra-faint satellites?}

The tidal stream's cross-section on the sky is normally a giveaway of the progenitor's mass. The low-density disrupting star clusters with small internal velocity dispersion $\sigma \lesssim 1 \mathrm{~km} \mathrm{~s}^{-1}$ typically produce tails that are only $\sim 0.1^{\circ}$ wide. On the other hand, a galaxy as massive as Sgr dwarf with its current $\sigma \lesssim 20 \mathrm{~km} \mathrm{~s}^{-1}$ (see e.g. Ibata et al., 2009) gives rise to streams that are at least $10^{\circ}$ across (see Figure 4 for example). This rule of thumb of course assumes comparable distances to the tidal tails and not hugely different dynamical ages. Depending on how aspherical the gravitational potential of the Galaxy is and how long ago the debris were stripped, even originally narrow tails can puff up with time.

Amongst the panoply of stellar substructure recently discovered in the Galactic halo, there are at least two streams that seem to occupy the parameter space intermediate between the star clusters and dwarf galaxies. These are the Orphan stream (Belokurov et al., 2006b, 2007a; Grillmair, 2006a) visible in Figure 4 as almost vertical streak of orange color crossing the Sgr debris at around $140^{\circ}<\mathrm{RA}<160^{\circ}$, and the Styx stream (Grillmair, 2009), the faint blue nebulous smear running at almost constant $\mathrm{Dec}=30^{\circ}$ from $\mathrm{RA}=250^{\circ}$ to $\mathrm{RA}=220^{\circ}$ where it starts to drop in Dec towards the Sgr stream. Curiously, both Orphan and Styx run in a close vicinity of the several of the Galactic ultra-faint satellites. The sky projection of the orbit of the Orphan stream takes it right through the position of the UMa II dwarf. The feasibility of such association is explored in Fellhauer et al. (2007) who conclude that UMa II could well be the stream's progenitor. However, as convincingly shown in Newberg et al. (2010), the early tentative estimates of the stream's radial velocity were incorrect and that the actual orbit of the stream is much more consistent with the 4D location of Segue 1. As regards to the Styx stream, when tracing its debris to the lower RA, Grillmair (2009) discovers a pronounced stellar clump within the stream's path. Dubbed Bootes III and subsequently confirmed with spectroscopy (Carlin et al., 2009) this is the most diffuse of all ultra-faints found so far.

\subsubsection{Broad and Invisible}

As the proper motion, spectroscopy and the variability wide-area surveys slowly catch up with the rapidly advancing sky imaging campaigns, it is possible to gauge the presence of stealth stellar structures, so diffuse that they elude detection in stellar halo maps akin to those described above. These detections are reminiscent of the original discovery of the Sgr dwarf (Ibata 
et al., 1994) that is too faint and spread out to be seen on a photographic plate but produces a booming signal in radial velocities.

Trinagulum-Andromeda is an extended stellar structure located at several tens of kpc from the Galactic centre (Rocha-Pinto et al., 2004). It is initially picked up as a faint excess of 2MASS M-giant stars, and later confirmed with the help of proper motion data and follow-up spectroscopy. As judged by the radial velocities of its members, the Tri-And cloud seems to be connected to the Southern Galactic counterpart of the Monoceros stream, and thus forms the more distant wraps of the Galactic Anti-centre Stellar Structure (Newberg et al., 2002; Ibata et al., 2003, Rocha-Pinto et al., 2003, Yanny et al., 2003). Majewski et al. (2004) and Martin et al. (2007b) report the detection of the Main Sequence stars in the Tri-And cloud, thus ridding of the last shreds of doubts as to the reality of its existence. Curiously, the recently discovered ultra-faint satellite Segue 2 (Belokurov et al., 2009) appears immersed in the debris of what very well might be the Tri-And cloud.

The recently discovered Cetus Polar Stream (Newberg et al., 2009) has avoided detection thanks to its low density and the overlap in projection with much brighter Sagittarius trailing stream. However, taking advantage of the SDSS spectroscopy available over a large portion of the Southern Galactic sky, Newberg et al. (2009) present a convincing argument in favor of a distinct stellar sub-structure, colder and more metal-poor than the Sgr debris. Koposov et al. (2012) provide the first sky map of the Cetus Polar Stream debris, and having obtained accurate measurements of the stream's distance and velocity gradients they argue that the sense of direction of the orbital motion of the CPS is opposite to that of Sgr. In their maps, the structure appears to be at least $20^{\circ}$ wide and some $40^{\circ} \mathrm{long}$, yet with only 0.1 mag width along the line of sight.

The charting of the Galactic halo at distances beyond $50 \mathrm{kpc}$ has been somewhat sluggish due to the obvious lack of suitably bright tracers covering a large enough area of the sky. A small fraction of the SDSS footprint, so-called Stripe 82 has been imaged repeatedly during the Supernovae campaign. Watkins et al. (2009) explores this multi-epoch dataset to identify RR Lyra stars. They find a significant over-density of RR Lyrae in the constellation of Pisces at galacto-centric distances of $D \sim 90 \mathrm{kpc}$, thus discovering the most distant sub-structure known in the Milky Way halo. Sesar et al. (2010a) confirm the discovery with a more sophisticated analysis of the same SDSS data, while Kollmeier et al. (2009); Sesar et al. (2010b) present the spectroscopic confirmation of the structure by obtaining velocities for several RR 
Lyra members. As of today the true extent of the Pisces Over-density is not known, but from the distribution of the RR Lyrae it subtends at least $10^{\circ}$ on the sky making it some $15 \mathrm{kpc}$ wide.

\subsection{Quantifying the amount of sub-structure}

Within the $\Lambda$ CDM paradigm, the global properties of the Galactic stellar halo, namely the total luminosity, the shape, the radial profile as well as the amount of sub-structure are simply the consequences of the Milky Way's accretion history and as such all have a straightforward interpretation. Observationally, however, these properties are awkward to pinpoint. For example, to gauge the flattening and the shape of the radial density profile, data across large portions of the Northern and the Southern Galactic sky are required. With pencil-beam surveys, the halo flattening or, more generally any deviation from spherical symmetry (e.g. triaxliaity), is impossible to determine and there is always a good chance of hitting unknowingly a stellar stream or a cloud, hence biasing the estimates of the density profile. Yet, in photometric studies, a robust global density model is vital when quantifying the amount of sub-structure. As the density distribution in the 6D phase-space, where the individual accreted fragments are readily identifiable, is collapsed onto the 3 spatial dimensions (or sometimes 2.5 or 2), the signal is diluted as a result of super-position of many structures. Therefore, even a small bias in the background properties can affect dramatically the amplitude of sub-structure. Of course, the "background" itself, in this picture, is nothing else but the stellar debris jumbled up more efficiently. Accordingly, the global law parameterizing the behavior of the background provides crucial information in which the mass of the satellites contributing to it and the time of their accretion is encoded.

\subsubsection{Spatial inhomogeneities}

With plenty of deep multi-band photometry in both Galactic hemispheres, the SDSS is an ideal resource to use to infer the global properties of such an immense structure as the Milky Way's stellar halo. A series of fits to the principal Galactic components as traced by the MS stars in the SDSS DR5 is presented in Jurić et al. (2008). This sample is dominated by the faint MS dwarfs and, therefore, can not trace the volume density in the Milky Way much further than $20 \mathrm{kpc}$. Within this radius, the halo appears to be well described by a single power law density model with the index $n \sim 2.8$. Importantly, this study confirms earlier indications of a substantial vertical 
flattening of the stellar halo $q \sim 0.6$. The results of Jurić et al. (2008) are corroborated by the modelling of the SDSS DR8 data with increased Southern Galactic hemisphere coverage published by Bonaca et al. (2012b). An attempt to delve deeper into the stellar halo can be found in Bell et al. (2008), where a simple color-cut (similar to that illustrated in the right panel of Figure 3p is used to isolate the brightest of the old MS stars in the halo. Using these blue, metal-poor turn-off stars, with typical $M_{g} \sim 4$, it is possible to discern halo structures as far as 30-40 kpc away from the Sun. However, as explained in Section 3.3, the spread in the intrinsic luminosities of the stars selected is as large as 3 magnitudes. There are two important consequences of such blurred vision. First, convolving the stellar halo distribution with large non-Gaussian errors in tracer distances can have strong destructive effects on the accuracy of the volume density inference. Second, when estimating the amplitude of small scale deviations from the background, a debris at one particular distance appears in several apparent magnitude bins (and hence distances), thus biasing high the total amount of sub-structure across the range probed. This effect is exacerbated at magnitudes close to the survey limit, as well as for stars with different age and/or metallicity.

While troubled by a number of issues outlined above, the analysis of Bell et al. (2008) is the first of its kind. Taking advantage of the impressive sky coverage and depth of the SDSS imaging, they provide a quantitative interpretation of the inhomogeneous stellar halo glimpsed by the earlier works. The main conclusions of the study by Bell et al. (2008) are as follows. First, a smooth density model for the MSTO tracers within $40 \mathrm{kpc}$ is not appropriate for the Milky Way halo, with most of the model parameters poorly constrained (see their Figures 4, 7 and 9). Second, even after excising the major known debris pile-ups such as Sagittarius stream and Virgo overdensity, the amount of sub-structure $\sigma /$ total, parameterized in terms of the scaled rms deviation $\sigma$ of the data around the smooth model, stays just under $40 \%$ from $r \sim 19$ mag to $r \sim 22 \mathrm{mag}$. In the presence of these large stellar halo structures, the $\sigma$ /total statistic grows with apparent magnitude (roughly proportional to distance) and reaches $>50 \%$ at $r \sim 21.5$. Finally, Bell et al. (2008) compare the values of $\sigma /$ total for the Milky Way halo traced by faint metal-poor MSTO stars in the SDSS to those obtained for the semi-analytic stellar halo simulations of the Galaxy by Bullock and Johnston (2005). The 11 model halos are made entirely of accreted stars, and show a minimal level of sub-structure $\sigma /$ total $>20 \%$. Accordingly, the final verdict is: the amount of sub-structure in the Galactic halo matches that in the hierarchical galaxy 
formation models, and, therefore, satellite accretion is the primary mode of the Milky Way's halo creation.

Helmi et al. (2011) aims to improve the analysis of Bell et al. (2008) by i) coming up with a more robust sub-structure quantification, and ii) comparing the SDSS data to the most recent stellar halo simulations. Similarly to Bell et al. (2008) they measure the stellar density scatter in bins of apparent magnitude and the two celestial coordinates. However, instead of calculating the amount of residual deviation between the data and the best-fit smooth parametric model, Helmi et al. (2011) work out the RMS around the mean stellar density in the bin. Predictably, the amount of sub-structure computed in this fashion is lower compared to that obtained by Bell et al. (2008), albeit only slightly. According to Helmi et al. (2011), across the apparent magnitude range of $18.5<r<22.5$, the normalized scatter $\operatorname{rms}(\rho) /<\rho>$ in the SDSS DR7 MSTO star density is at the level of $30 \%$ to $40 \%$. These rather serious levels of inhomogeneity found in the SDSS data nonetheless appear low when contrasted with the degree of sub-structure in simulated stellar halos. For the comparison with the data, Helmi et al. (2011) examine the smoothness of the mock stellar halos produced by Cooper et al. (2010). These are built into the Aquarius DM-only halos (Springel et al., 2008) by tagging $1 \%$ of the most-bound particles in selected sub-halos and following them to redshift 0. Compared to the mock "Milky Ways" of Bullock and Johnston (2005), these have the obvious advantages of being fabricated in the Cosmological setting, and with a superior resolution. However, there are disadvantages too. First, the Aquarius suite explores only half as many accretion histories, in fact, in the end, there are only 4 stellar halos analyzed in Helmi et al. (2011), compared to 11 in Bullock and Johnston (2005). Second, these Galaxy analogs do not posses disks. A quick glance at the Figure 5 of Helmi et al. (2011) reveals: all stellar halos of Cooper et al. (2010) are highly irregular, with $50 \%<\frac{\operatorname{rms}(\rho)}{\langle\rho\rangle}<150 \%$. The authors raise concern that some of the data-model discrepancy could be due to the combined effects of the MSTO sample contamination and the presence of a smooth, in-situ formed stellar halo component. Nonetheless, they conclude that there exists considerable tension between the observations of the Galactic stellar halo sub-structure and the predictions of the simple but high-resolution model. Even though the halos of both the real and the mock Galaxy are very inhomogeneous, the simulations easily reach 2-3 times the observed scatter on scales as small as few degrees. 

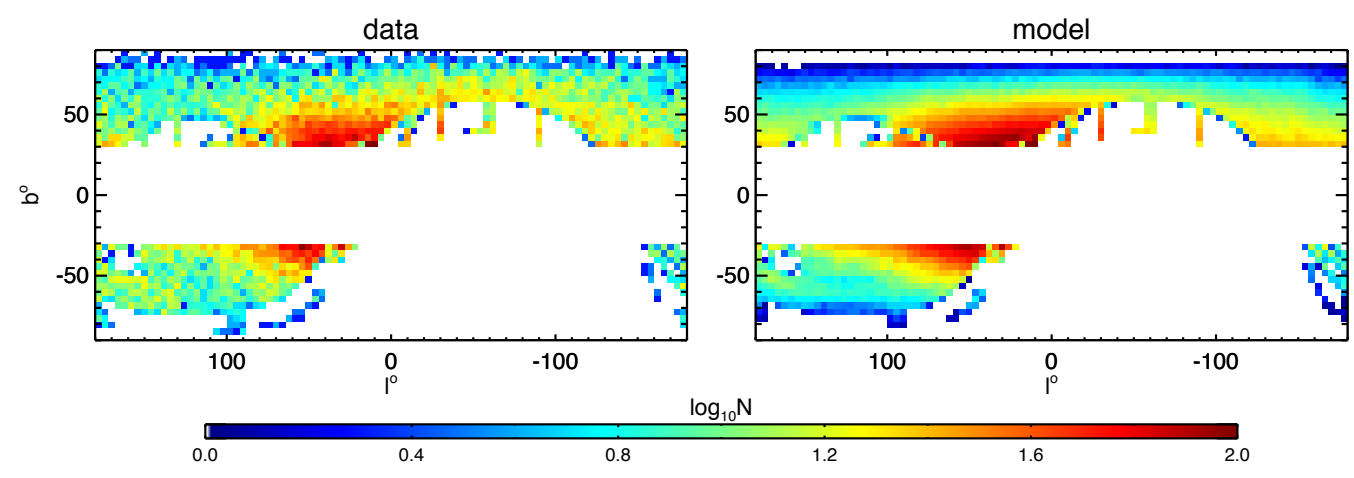

Figure 7: Stellar halo of the Milky Way traced by the BHB stars. Left Distribution of the SDSS DR8 BHB candidates in the Galactic $l$ and $b$. Right Best-fit model of the stellar halo density distribution shown in the Left panel, from Deason et al. (2011). The model halo is flattened with $q \sim 0.6$ and has a break in the radial density profile at $r \sim 27 \mathrm{kpc}$ where the power-law index changes from -2.3 to -4.6 . Figure courtesy of Alis Deason, IoA/UCSC.

The picture of the utter chaos in the inner parts of the Galactic stellar halo is re-visited in Deason et al. (2011). Instead of using the more abundant MSTO stars, they choose to model the halo volume density with Blue Horizontal Branch stars. While these stars are rarer, their higher intrinsic luminosities, lower levels of contamination and accurate absolute magnitude calibration independent of age and chemistry all make these a better fit for the task. There are, nonetheless, several limitations to the use of BHBs as tracers. For example, being some $\sim 4$ magnitudes brighter and at least two orders of magnitude less frequent as MSTO, these come in particularly low numbers at bright apparent magnitudes due to the size of the volume probed. Additionally, while their blue color makes them stand out dramatically compared to most other stellar populations at high Galactic latitudes, there is one troublesome impostor. Blue Stragglers (see Figure 2) have close to identical broad-band colors but are $\sim 1.5$ mag fainter. Outnumbering the BHBs by a factor of 2 on average, these may pose a serious problem by scrambling the tracer counts as a function of apparent magnitude. Deason et al. (2011) solve both the problem of the limited dynamic range and of the contamination by including the BS stars in the model. For all "blue" stars in the SDSS DR8, i.e. $-0.25<g-r<-0$, the probability of belonging to the BHB or the BS population is assigned based on their $u-g$ and $g-r$ colors. As a result, the number density of stars in volume elements of the space spanned by position of the sky, color and apparent magnitude can be modeled sim- 
ply as the sum of the contributions from BHBs and BSs, weighted by their conditional probabilities.

The results of the maximum-likelihood analysis presented in Deason et al. (2011) are summarized for the impatient reader in the article's title "Squashed, broken but smooth". In other words: out to $40 \mathrm{kpc}$, the Galactic stellar halo appears to be highly flattened, the density profile follows closely the broken power law and, most interestingly, the overall level of sub-structure detected using the BHB tracers is rather low. At small and intermediate distances, $\sigma /$ total rises from as low $10 \%$ to at most $20 \%$ irrespective of the spatial scale of density perturbations. At large distance, $\sigma$ /total is close to $20 \%$ on most scales, but rises to $40 \%$ for the angular sizes of several hundreds of degrees. These numbers are obtained by excluding from the modeling the regions of the sky with known large-scale halo overdensities. Even when these are included, the small-size inhomogeneities are only $10 \%<\sigma /$ total $<30 \%$. While, superficially, these estimates differ significantly from those quoted in Bell et al. (2008), there are several possible solutions to this discrepancy. Both methods have their weak points. It is quite likely that some of the halo mess observed by Bell et al. (2008) is simply due to the limitations of the MSTO stars as tracers. On the other hand, the average number of BHBs in a $1^{\circ} \times 1^{\circ}$ pixel is small, hence limiting the areas of the sky tested by Deason et al. (2011) to those towards the inner Galaxy where mixing is more efficient. On slightly larger angular scales (several degrees or so), it is, however, safe to conclude that the inner stellar halo is indeed smooth.

\subsubsection{Phase-space sub-structure. Spaghetti, ECHOS and SKOs}

As it is much easier to identify the accreted satellite debris in the phasespace compared to simple sky density maps or 3D spatial maps, several attempts have been made to search for the surviving Galactic sub-structure in the datasets of wide area spectroscopic surveys. The Spaghetti survey (e.g. Morrison et al., 2000) is the first brave endeavor to collect substantial numbers of genuine halo tracers in a large distance range. It is set up to gather photometry and the follow-up spectroscopy in several tens of "pencil-beam" fields over the area covering many tens of degrees. The analysis dealing with the quantification of the presence of sub-structure in the final set of 101 giants with spectra covering distances up to $100 \mathrm{kpc}$ is presented in Starkenburg et al. (2009). They report the detection of 1 group and 6 pairs of clumped stars and conclude that their findings of $10 \%$ of sub-structure in the halo are consistent with the accretion scenarios in which early and/or massive 
satellite infall leads to the creation of broad phase-space features.

The SEGUE survey that has taken $\sim 240,000$ spectra in $>200$ pointings spread over $\sim 11,000$ square degrees is the ideal source of data to carry out a systematic search for un-relaxed sub-structure. Schlaufman et al. (2009) do exactly that, and detect in 137 lines of sight studied 10 high-confidence ECHOS, elements of cold halo substructure as traced by metal-poor MSTO stars with distances in the range $10<D(\mathrm{kpc})<20$. As the ECHOS identification algorithm described is automated, the work also contains the results of the completeness calculation for the sub-structure search in the velocity space. These are then used to turn the numbers of detections into the predictions of sub-structure fractions existent in the halo: they conclude that within $1 / 3$ of the volume of the Galactic halo, there ought to be of the order of $10 \%$ of ECHOS.

Xue et al. (2011) use the spectroscopic sample of $\sim 4$, 000 SDSS BHB stars to gauge the power spectrum of the kinematic sub-structure in the stellar halo by measuring the excess of close stellar pairs whose relative distances are measured in the 4D comprised of 3 spatial coordinates and the line-of-sight velocity. The presence of sub-structure is clearly detected when the observed close-pair distribution is compared to that of the mock smooth halo created by scrambling the heliocentric distances of the SDSS BHBs. Moreover, as predicted, the outer halo $(20<D<40 \mathrm{kpc})$ exhibits a stronger sub-structure signal as compared to the inner one $(5<D<20 \mathrm{kpc})$. However, when comparing to the simulations of Bullock and Johnston (2005), Xue et al. (2011) find appreciably less signal overall, in particular, at the intermediate $4 \mathrm{D}$ distance scales.

Finally, at moderate distances from the Sun, relatively accurate stellar proper motion measurements have been available for some time. Combined with photometric parallax, this opens up the possibility to calculate all 6 of the phase-space coordinates. Accordingly, Smith et al. (2009) exploit the accurate proper motions calculated by Bramich et al. (2008) using the multi-epoch data in the SDSS Stripe 82 region, to compile a catalog of $\sim$ 1,700 MS sub-dwarfs within $5 \mathrm{kpc}$ from the Sun with full space velocities and $3 \mathrm{D}$ coordinates measured. In estimating the tangential components of the stars velocity, the main source of error is the distance uncertainty, which is actually present at the rather modest level of 10\%. After subtracting a smooth Galaxy model from the distribution of the angular momenta $J_{z}, J_{\perp} 3$ significant clumps are detected, one of which has been previously discovered in the pioneering work by Helmi et al. (1999). 


\section{Putting the puzzle together}

It is obvious why in a framework which builds the Universe from bottom up, the smallest galaxies are under double scrutiny: such structure formation paradigm risks losing its credibility if its basic blocks look unlifelike. For $\Lambda \mathrm{CDM}$, predicting the properties of the local dwarf galaxies, while seemingly completely straightforward, has turned out to be a trying quest. As this review argues, before a faithful comparison between the dwarf galaxies formed according to the Cold Dark Matter model and the observed satellites can be carried out, a number of loose ends need to be tied up. First, the host-to-host variations in the properties of the DM sub-halo populations due to the parent halo accretion history and the environment need to be quantified. Also, it is not an overstatement to say that our understanding of the baryonic processes to do with cooling and feedback, although developing swiftly, is not yet mature and, thus deserves further improvement. Observationally, it seems now possible to provide better measurements of the basic properties of the host such as the Galactic total mass, the mass of the disk and, possibly even the concentration of the DM halo. Additionally, as the quantitative comparison is presently based around the satellite luminosity function, it is crucial to figure out the genesis of the smallest objects dominating the census, the ultra-faints. Finally, it is clear that the same DM and baryonic physical processes that have sculpted the $z=0$ dwarf satellite population are also responsible for the creation of the stellar halo. Therefore, a successful galaxy formation model is expected to get both the survived and the perished satellites right.

\subsection{Light Galactic DM halo with high concentration}

Deason et al. (2012b) tackle the issue of the dearth of reliable tracers in the outer halo. They take advantage of the availability of the multiple epoch imaging data across the SDSS field of view. Due to the survey geometry, a considerable number of imaging "stripes" overlap (mostly around the survey poles) and the photometry from the multiple runs can be combined to yield higher signal-to-noise magnitude measurements. This is especially valuable for the faint BHB candidate stars, whose single-epoch $u$-band magnitudes are simply too unreliable for the conventional broad-band BHB/MS/MSTO classification. Using the overlaps as well as the multi-epoch data from the SDSS Stripe 82, Deason et al. (2012b) find 43 distant BHB candidates with $20<g<22$ and follow these up with deep spectroscopy on the ESO's VLT. 
Once a handful the QSO interlopers are removed, of the remaining 38 Acolored stars, 7 are genuine BHBs in the distance range $80-150 \mathrm{kpc}$. Note that, at these faint magnitudes, even the remaining 31 BS stars populate the poorly explored regime between 30 and $90 \mathrm{kpc}$. Their final sample also contains 8 cool carbon stars that span a distance range 80 to $160 \mathrm{kpc}$. This combined spectroscopic sample is the largest collection to date of the halo tracers with distances beyond $60 \mathrm{kpc}$. Curiously, outside $100 \mathrm{kpc}$ from the Galactic center, the line-of-sight velocity dispersion plummets to a rather low 50-60 $\mathrm{km} \mathrm{s}^{-1}$. Unless the stellar tracers considered have a significant tangential bias and/or their density drops much faster than the already rather steep power law with index -4.5 , this measurement implies the mass of the Milky Way in the range of $5-10 \times 10^{11} M_{\odot}$ within $150 \mathrm{kpc}$.

To figure out how concentrated the DM halo of the Galaxy is, the total matter density has to be mapped out in a sufficiently wide range of distances around or beyond the Solar neighborhood (inside $R_{0}$, the disk dominates the mass budget). Deason et al. (2012a) exploit the sheer volume probed by the SDSS BHB stars with known radial velocities to simultaneously constrain the halo velocity anistropy and the matter distribution. By approximating the gravitational potential of the Galaxy as a power-law and adopting the tracer density distribution pinned down in Deason et al. (2011), they find that the rotation curve of the Galaxy has to start falling appreciably already at around $30 \mathrm{kpc}$ from the center. They ague that the preferred value of the normalization and the power law index of the gravitational potential are inconsistent with massive, i.e. $2 \times 10^{12} M_{\odot}$ DM halos with concentrations around $c \sim 10$. Instead, a lighter and more concentrated, i.e. $c \sim 20$ dark halo is favored.

\subsection{Smooth stellar halo and signatures of early accretion}

The stellar masses in the known large-scale over-densities in the Galactic halo (see Section 4.1.2 sum up to the approximate total of $\sim 3 \times 10^{8} M_{\odot}$. This is to be compared with the estimates of the total stellar halo mass (e.g. Bell et al., 2008; Deason et al., 2011) of the order of $\sim 10 \times 10^{8} M_{\odot}$. Therefore, perhaps as much as $70 \%$ of the stellar halo within $40 \mathrm{kpc}$ is in a smooth or, more accurately, well-mixed, component, which can be described by a broken power-law density profile with a flattening of $q \sim 0.6$. On contrary, the Andromeda's stellar halo harbors no such feature in its density profile over twice as large range of distances, albeit it does not look nearly as smooth Gilbert 

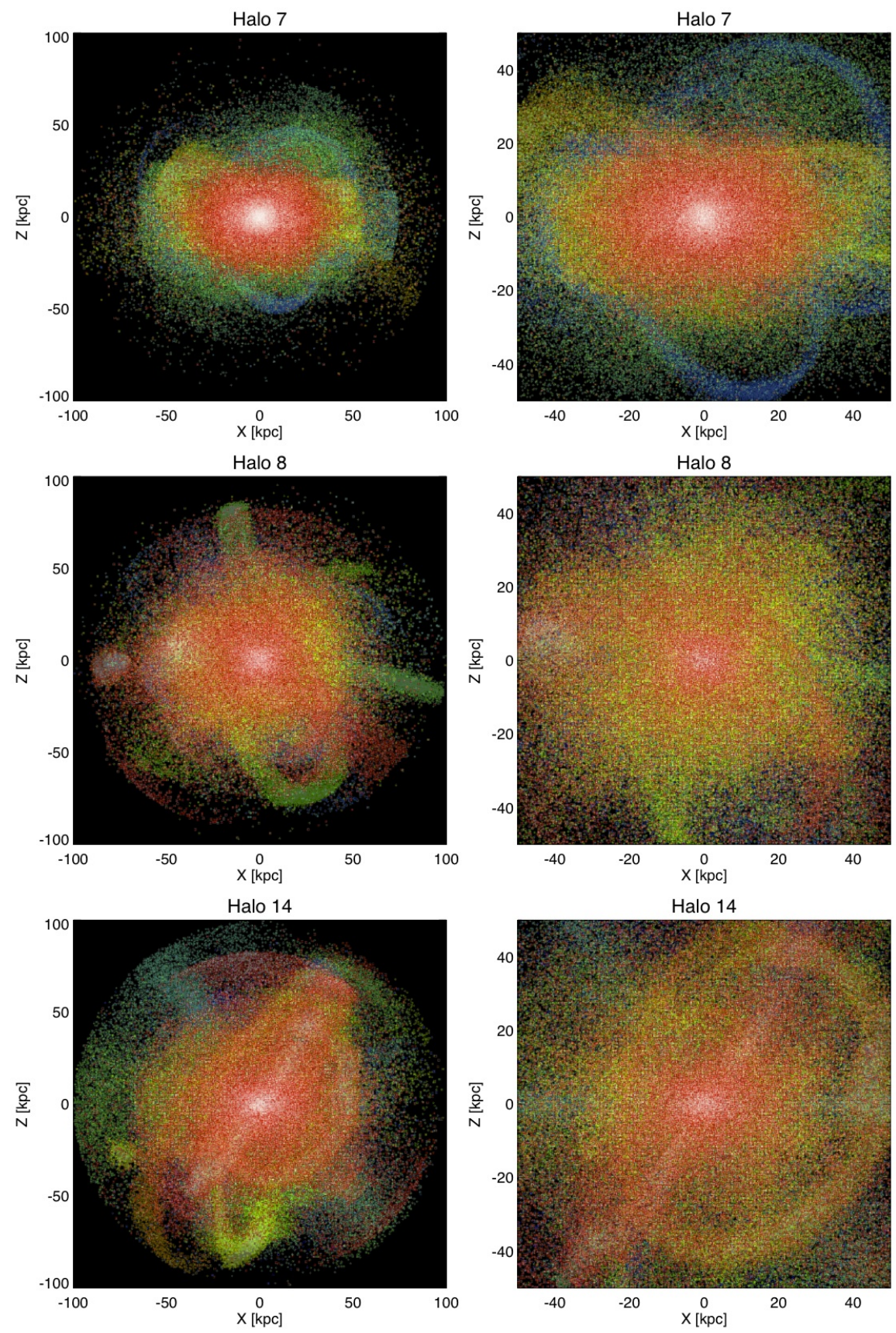

Figure 8: Three of the 11 Galactic stellar halos simulated by Bullock and Johnston (2005). Particles are color-coded according to the mass of the progenitor, with most massive in red and least massive in blue. The smooth, flattened Halo 7 has a density break at $24 \mathrm{kpc}$ and represents the simulation closest to the observed Galactic stellar halo. Halo 8 gives a clue of how the stellar halo in M31 might look like. 
et al., 2012). Deason et al. (2013) investigate whether stellar halos with density breaks similar to that of the Milky Way can be assembled purely through satellite accretion, and if yes, what controls the break prominence and the radius. By studying a set of $11 \mathrm{~N}$-body simulations published by Bullock and Johnston (2005) they come to the following conclusion. In the simulations studied, the radial profiles of stars from individual accretion events can be described by single power law, double power law or can be so ill-defined that neither of the simple models works. Ancient ( $>10^{\mathrm{Gyr}}{ }^{1}$ ) debris have had plenty of time to mix and therefore at $z=0$ the radial profile is comfortably fit with a single power-law. Old (7-10 Gyr) debris have spread out over a range of Galacto-centric distances but around the progenitor's apo-centre, the drop in stellar density remains. Recent $(<6$ Gyr) mergers have not yet filled the entire volume inward of the apo-centre and their radial distribution still peaks at $R>0$.

The stellar halo (in this model) is just a superposition of the debris from the individual events across the entire accretion history. The combined stellar profile can have a distinct break (at the average apo-centre of the most massive accreted satellites) only if the most significant merger(s) happened at the right time, i.e. 8-10 Gyr. Additionally, it is required to dampen the accretion rate at the subsequent epochs: as the Galaxy grows, the satellites that arrive with increasingly larger apo-centers thus can flatten out the density profile around and beyond the break radius, thus erasing this feature altogether. The hypothesis that the density break in the Galactic stellar halo reflects the apo-center(s) of the massive satellite(s) accreted at early epochs can be tested with 3D kinematics. Radial velocities of stars tend to zero around the apo-center of the orbit, therefore the radial velocity dispersion of the stellar halo should have a dip around the break radius as well as an increase in the tangential anisotropy. Moreover, there exists a potentially powerful diagnostic to decipher the properties of this old merger. Namely, if the metallicities of the stellar halo tracers around the break radius (i.e. $20<R<30 \mathrm{kpc}$ ) are available, then it is possible to distinguish between the accretion of one or two massive satellite(s) and the accretion of a group of dwarfs. If only one system contributed the bulk of the debris within the break then the radial velocity dispersion dip around the break radius should

\footnotetext{
${ }^{1}$ In this definition, the age marks the time of when the stars became unbound, which implies slightly earlier epochs for the arrival of the progenitor.
} 
be most visible in stars with the chemical abundance of that satellite. The density break created via superposition of the debris from many different satellites is not dominated by any particular stellar population, and hence, the drop in radial velocity dispersion should occur, albeit weakly, across the metallicity range.

Figure 8 shows the examples of three Galactic stellar halos created in simulations by Bullock and Johnston (2005). Here, the X-Z distributions of particles color-coded according to the mass of the progenitor (red for most massive, blue for the least massive) are shown for the inner $100 \mathrm{kpc}$ (left panel) and the inner $50 \mathrm{kpc}$ (right panel). The differences in the structures of Halo 7 and Halo 8 provide visual clues as to the findings of Deason et al. (2013). Halo 7 has a peaked accretion history, with the bulk of the stellar halo assembled quickly around $8 \mathrm{Gyr}$ ago. Within $40 \mathrm{kpc}$ from the center of the Galaxy, the stellar halo seems smooth, flattened and strongly aligned with the disk (compare to the view of the Galactic stellar halo in Figure 7). Note that Halo 7 has a prominent break in the radial density profile at $24 \mathrm{kpc}$, closely matching the observed properties of the Milky Way halo as traced by the BHBs. Halo 8, on contrary, shows no evidence of the break - this turns out to be a giveaway of its accretion history and the present day appearance. Halo 8 can be categorized by a continuous infall of satellites with three particularly massive fragments disrupting at 11, 7 and 1-2 Gyr. The stellar distribution is evidently more extended and substantially messier (even in the inner parts) as compared to that of the "quiet" Halo 7. Additionally, neither obvious vertical flattening nor alignment with the disk can be seen.

\subsection{Nature of the faintest of the ultra-faint satellites}

The absolute majority of the stellar populations in the ultra-faint dwarf satellites are as old as the oldest Galactic globular clusters and are similarly metal-poor, as revealed, for example, by their deep Color-Magnitude Diagrams. Given their low total stellar masses, the velocity dispersions in the range of $2<\sigma<9 \mathrm{~km} \mathrm{~s}^{-1}$ and the recently detected spreads in metallicity, there is now little doubt that these are the remnants of the galaxies born at high redshifts in low-mass DM halos. What is not yet completely clear is the exact mapping between the galaxies of a particular luminosity at $z=0$ and the original sub-halo mass at the epoch of formation. This is simply due to the fact that for such faint stellar systems, there is not enough stars currently available to trace the total matter distribution out to large distances from the center. Hence, even though their present-day central masses are 

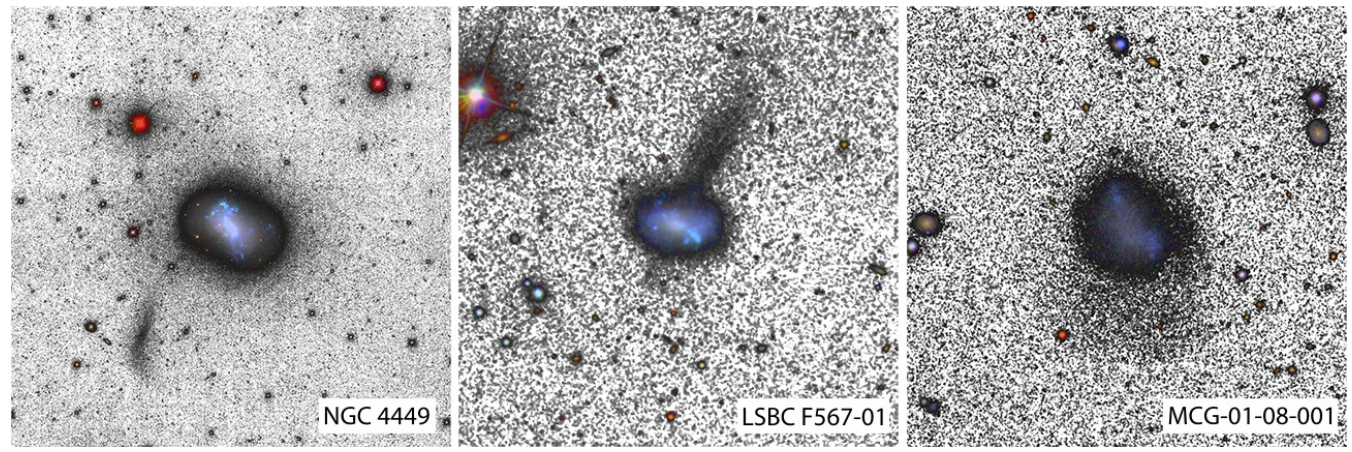

Figure 9: Dwarfs accreting dwarfs. This shows the SDSS image mosaics of the three Local Volume dwarf galaxies (with luminosities similar to that of the SMC) disrupting their satellites with masses as low as a hundredth of the host's. The images are processed to enhance the low-surface brightness features. From Left to Right: the dwarf-to-dwarf accretion sequence. The satellite of the NGC 4449 is showing the first signs of tidal interaction, the satellite of LSBC F567-01 is critically stretched, very close to being fully destroyed, the satellite of MCG-01-08-001 is taken apart and incorporated into the host, but still visible as faint stellar plume around the Southern edge of host's halo.

being constrained, the total mass and the extent of their DM halos is still an enigma.

All semi-analytic models of the dwarf galaxy formation predict that most satellites lose significant amounts of their dark and luminous matter to the host's tides. The precise mass loss is the crucial middle part in the model that has at one end a variety of dark hosts with the mass function steeply rising at low masses, and at the other, the observed population of Galactic survivors. Relying on the fact that the details of the tidal harassment are captured at the appropriate level in the high resolution N-body simulations, the luminosity function of the Milky Way satellites is inverted to reveal the physics of star formation in the early Universe. However, it is now apparent that the inclusion of the baryons (perhaps even as simple as adding a disk component to the host galaxy) can alter the satellite survival rates dramatically. Additionally, in the hierarchical Universe, the smallest satellites have a non-zero chance of being first accreted onto the more massive dwarf galaxies before finally merging with their "Milky Way" host. Such satellites of satellites with large host-to-satellite mass ratios have been known to exist in the galaxy formation simulations, but only now begin to be discovered in nature. For example, Martínez-Delgado et al. (2012) report the discovery of the early stages of the accretion of a low-luminosity dwarf by the galaxy as 
massive as the SMC, namely NGC 4449. Figure 9 shows this and two more examples of different stages of the accretion of dwarfs onto dwarfs.

To increase the dynamic range, the images in the three panels of Figure 9 are composed of two versions of the same SDSS mosaic image: the original one in color and the greyscale one obtained by applying the median filter to triples of pixels in the three SDSS filters to minimize the "patchwork" effects of the sky slightly misaligned between different SDSS runs. The original SDSS color image and the stretched and inverted greyscale image are then combined by choosing the pixel value to be the highest one between the two images. This simple image processing (also employed by e.g. MartínezDelgado et al., 2010, 2012) makes it possible to show simultaneously the central object where most of the light is as well as the further low surface brightness features that are to do with the ongoing tidal interactions. While the left panel shows the beginning of the process of accretion, the middle and the right panels of the Figure display more advanced stages of the dwarf infall and disruption. LSBC F567-01 in the center is seen taking apart a lowluminosity satellite which looks prominently stretched and perhaps close to its total disintegration. Finally, MCG-01-08-001 in the right panel, is clearly about to finish eating up a smaller satellite, which presently can only be seen as extended faint plume of stellar debris in the Southern parts of the host.

Taking into account the existing evidence of a likely association between objects like Segue 1, Segue 2, Bootes II and III and the known large-scale sub-structure in the Galactic stellar halo, the following scenario is perhaps possible. The faintest of the currently known Milky Way satellites were born as sub-systems of larger dwarfs and were subsequently pre-processed by their first hosts' tidal fields. They have probably lost much of their original mass but their remnant cores survived as part of the bigger dwarf galaxy until the whole system was accreted by the Milky Way. Their parent galaxies were sufficiently massive to be dragged into the inner Milky Way where they were destroyed and are visible today only as streams and clouds of stars in the halo. Some of the satellites of satellites persisted in the Milky Way halo and can now be observed as the faintest of the ultra-faint dwarfs. In this scheme, the current stellar and the DM masses are severely truncated: thanks to the pre-processing in the gravitational field of the parent dwarf their current luminosities could be much lower than what is attainable by the lowest mass dwarfs in the field. This in turn, would imply that the fast drop in the efficiency of the dwarf galaxy formation actually happens at the sub-halo masses that are higher than previously envisaged. Because the satellites 
with absolute magnitudes around $-2>M_{V}>-4$ can only (or mostly) exist as part of bigger dwarf systems, their distribution in the Galaxy is different from that of the accreted field dwarf population. Their radial density profile should be strongly radially concentrated due to the combination of the two effects. First, their parent galaxies were massive enough to end up close to the center due to the dynamical friction. Second, in the Galaxy most of the large systems (apart from the Sgr dwarf) were accreted as early as 8-10 Gyr, when the mass and the virial mass of the Milky Way were much smaller. Taking these effects into account, much lower numbers of satellites as faint as Segue I or II are predicted to be discovered by the future deep all sky surveys. 


\section{Acknowledgments}

V. Belokurov thanks The Royal Society the support. The work on this review has received funding from the European Research Council under the European Union's Seventh Framework Programme (FP/2007-2013) / ERC Grant Agreement n. 308024. The author has enjoyed conversations with A. Deason, W. Evans, A. Helmi, M. Irwin, S. Koposov, P. Kroupa, J. Norris, M. Smith, E. Starkenburg and E. Tolstoy. 


\section{References}

Ahn, C. P., Alexandroff, R., Allende Prieto, C., Anderson, S. F., Anderton, T., Andrews, B. H., Aubourg, É., Bailey, S., Balbinot, E., Barnes, R., et al., Dec. 2012. The Ninth Data Release of the Sloan Digital Sky Survey: First Spectroscopic Data from the SDSS-III Baryon Oscillation Spectroscopic Survey. ApJS 203, 21.

Aihara, H., Allende Prieto, C., An, D., Anderson, S. F., Aubourg, É., Balbinot, E., Beers, T. C., Berlind, A. A., Bickerton, S. J., Bizyaev, D., Blanton, M. R., Bochanski, J. J., Bolton, A. S., Bovy, J., Brandt, W. N., Brinkmann, J., Brown, P. J., Brownstein, J. R., Busca, N. G., Campbell, H., Carr, M. A., Chen, Y., Chiappini, C., Comparat, J., Connolly, N., Cortes, M., Croft, R. A. C., Cuesta, A. J., da Costa, L. N., Davenport, J. R. A., Dawson, K., Dhital, S., Ealet, A., Ebelke, G. L., Edmondson, E. M., Eisenstein, D. J., Escoffier, S., Esposito, M., Evans, M. L., Fan, X., Femenía Castellá, B., Font-Ribera, A., Frinchaboy, P. M., Ge, J., Gillespie, B. A., Gilmore, G., González Hernández, J. I., Gott, J. R., Gould, A., Grebel, E. K., Gunn, J. E., Hamilton, J.-C., Harding, P., Harris, D. W., Hawley, S. L., Hearty, F. R., Ho, S., Hogg, D. W., Holtzman, J. A., Honscheid, K., Inada, N., Ivans, I. I., Jiang, L., Johnson, J. A., Jordan, C., Jordan, W. P., Kazin, E. A., Kirkby, D., Klaene, M. A., Knapp, G. R., Kneib, J.-P., Kochanek, C. S., Koesterke, L., Kollmeier, J. A., Kron, R. G., Lampeitl, H., Lang, D., Le Goff, J.-M., Lee, Y. S., Lin, Y.-T., Long, D. C., Loomis, C. P., Lucatello, S., Lundgren, B., Lupton, R. H., Ma, Z., MacDonald, N., Mahadevan, S., Maia, M. A. G., Makler, M., Malanushenko, E., Malanushenko, V., Mandelbaum, R., Maraston, C., Margala, D., Masters, K. L., McBride, C. K., McGehee, P. M., McGreer, I. D., Ménard, B., Miralda-Escudé, J., Morrison, H. L., Mullally, F., Muna, D., Munn, J. A., Murayama, H., Myers, A. D., Naugle, T., Neto, A. F., Nguyen, D. C., Nichol, R. C., O'Connell, R. W., Ogando, R. L. C., Olmstead, M. D., Oravetz, D. J., Padmanabhan, N., Palanque-Delabrouille, N., Pan, 
K., Pandey, P., Pâris, I., Percival, W. J., Petitjean, P., Pfaffenberger, R., Pforr, J., Phleps, S., Pichon, C., Pieri, M. M., Prada, F., Price-Whelan, A. M., Raddick, M. J., Ramos, B. H. F., Reylé, C., Rich, J., Richards, G. T., Rix, H.-W., Robin, A. C., Rocha-Pinto, H. J., Rockosi, C. M., Roe, N. A., Rollinde, E., Ross, A. J., Ross, N. P., Rossetto, B. M., Sánchez, A. G., Sayres, C., Schlegel, D. J., Schlesinger, K. J., Schmidt, S. J., Schneider, D. P., Sheldon, E., Shu, Y., Simmerer, J., Simmons, A. E., Sivarani, T., Snedden, S. A., Sobeck, J. S., Steinmetz, M., Strauss, M. A., Szalay, A. S., Tanaka, M., Thakar, A. R., Thomas, D., Tinker, J. L., Tofflemire, B. M., Tojeiro, R., Tremonti, C. A., Vandenberg, J., Vargas Magaña, M., Verde, L., Vogt, N. P., Wake, D. A., Wang, J., Weaver, B. A., Weinberg, D. H., White, M., White, S. D. M., Yanny, B., Yasuda, N., Yeche, C., Zehavi, I., Apr. 2011. The Eighth Data Release of the Sloan Digital Sky Survey: First Data from SDSS-III. ApJS 193, 29.

Alexander, P. E. R., Gieles, M., May 2013. Constraining the initial conditions of globular clusters using their radius distribution. MNRAS 432, L1.

An, D., Johnson, J. A., Clem, J. L., Yanny, B., Rockosi, C. M., Morrison, H. L., Harding, P., Gunn, J. E., Allende Prieto, C., Beers, T. C., Cudworth, K. M., Ivans, I. I., Ivezić, Ž., Lee, Y. S., Lupton, R. H., Bizyaev, D., Brewington, H., Malanushenko, E., Malanushenko, V., Oravetz, D., Pan, K., Simmons, A., Snedden, S., Watters, S., York, D. G., Dec. 2008. Galactic Globular and Open Clusters in the Sloan Digital Sky Survey. I. Crowded-Field Photometry and Cluster Fiducial Sequences in ugriz. ApJS $179,326-354$.

Battaglia, G., Helmi, A., Morrison, H., Harding, P., Olszewski, E. W., Mateo, M., Freeman, K. C., Norris, J., Shectman, S. A., Dec. 2005. The radial velocity dispersion profile of the Galactic halo: constraining the density profile of the dark halo of the Milky Way. MNRAS 364, 433-442.

Bell, E. F., Xue, X. X., Rix, H.-W., Ruhland, C., Hogg, D. W., Dec. 2010. Stellar Population Variations in the Milky Way's Stellar Halo. AJ 140, 1850-1859.

Bell, E. F., Zucker, D. B., Belokurov, V., Sharma, S., Johnston, K. V., Bullock, J. S., Hogg, D. W., Jahnke, K., de Jong, J. T. A., Beers, T. C., Evans, N. W., Grebel, E. K., Ivezić, Ž., Koposov, S. E., Rix, H.-W., Schneider, 
D. P., Steinmetz, M., Zolotov, A., Jun. 2008. The Accretion Origin of the Milky Way's Stellar Halo. ApJ 680, 295-311.

Bellazzini, M., Correnti, M., Ferraro, F. R., Monaco, L., Montegriffo, P., Feb. 2006. The age of the main population of the Sagittarius dwarf spheroidal galaxy. Solving the "M giant conundrum". A\&A 446, L1-L4.

Belokurov, V., Evans, N. W., Bell, E. F., Irwin, M. J., Hewett, P. C., Koposov, S., Rockosi, C. M., Gilmore, G., Zucker, D. B., Fellhauer, M., Wilkinson, M. I., Bramich, D. M., Vidrih, S., Rix, H.-W., Beers, T. C., Schneider, D. P., Barentine, J. C., Brewington, H., Brinkmann, J., Harvanek, M., Krzesinski, J., Long, D., Pan, K., Snedden, S. A., Malanushenko, O., Malanushenko, V., Mar. 2007a. The Hercules-Aquila Cloud. ApJL 657, L89-L92.

Belokurov, V., Evans, N. W., Irwin, M. J., Hewett, P. C., Wilkinson, M. I., Jan. 2006a. The Discovery of Tidal Tails around the Globular Cluster NGC 5466. ApJL 637, L29-L32.

Belokurov, V., Evans, N. W., Irwin, M. J., Lynden-Bell, D., Yanny, B., Vidrih, S., Gilmore, G., Seabroke, G., Zucker, D. B., Wilkinson, M. I., Hewett, P. C., Bramich, D. M., Fellhauer, M., Newberg, H. J., Wyse, R. F. G., Beers, T. C., Bell, E. F., Barentine, J. C., Brinkmann, J., Cole, N., Pan, K., York, D. G., Mar. 2007b. An Orphan in the "Field of Streams". ApJ 658, 337-344.

Belokurov, V., Koposov, S. E., Evans, N. W., Peñarrubia, J., Irwin, M. J., Smith, M. C., Lewis, G. F., Gieles, M., Wilkinson, M., Gilmore, G., Olszewski, E. W., Niederste-Ostholt, M. N., Jan. 2013. Precession of the Sagittarius stream. ArXiv e-prints.

Belokurov, V., Walker, M. G., Evans, N. W., Faria, D. C., Gilmore, G., Irwin, M. J., Koposov, S., Mateo, M., Olszewski, E., Zucker, D. B., Oct. 2008. Leo V: A Companion of a Companion of the Milky Way Galaxy? ApJL 686, L83-L86.

Belokurov, V., Walker, M. G., Evans, N. W., Gilmore, G., Irwin, M. J., Just, D., Koposov, S., Mateo, M., Olszewski, E., Watkins, L., Wyrzykowski, L., Mar. 2010. Big Fish, Little Fish: Two New Ultra-faint Satellites of the Milky Way. ApJL 712, L103-L106. 
Belokurov, V., Walker, M. G., Evans, N. W., Gilmore, G., Irwin, M. J., Mateo, M., Mayer, L., Olszewski, E., Bechtold, J., Pickering, T., Aug. 2009. The discovery of Segue 2: a prototype of the population of satellites of satellites. MNRAS 397, 1748-1755.

Belokurov, V., Zucker, D. B., Evans, N. W., Gilmore, G., Vidrih, S., Bramich, D. M., Newberg, H. J., Wyse, R. F. G., Irwin, M. J., Fellhauer, M., Hewett, P. C., Walton, N. A., Wilkinson, M. I., Cole, N., Yanny, B., Rockosi, C. M., Beers, T. C., Bell, E. F., Brinkmann, J., Ivezić, Ž., Lupton, R., May 2006b. The Field of Streams: Sagittarius and Its Siblings. ApJL 642, L137-L140.

Belokurov, V., Zucker, D. B., Evans, N. W., Kleyna, J. T., Koposov, S., Hodgkin, S. T., Irwin, M. J., Gilmore, G., Wilkinson, M. I., Fellhauer, M., Bramich, D. M., Hewett, P. C., Vidrih, S., De Jong, J. T. A., Smith, J. A., Rix, H.-W., Bell, E. F., Wyse, R. F. G., Newberg, H. J., Mayeur, P. A., Yanny, B., Rockosi, C. M., Gnedin, O. Y., Schneider, D. P., Beers, T. C., Barentine, J. C., Brewington, H., Brinkmann, J., Harvanek, M., Kleinman, S. J., Krzesinski, J., Long, D., Nitta, A., Snedden, S. A., Jan. 2007c. Cats and Dogs, Hair and a Hero: A Quintet of New Milky Way Companions. ApJ 654, 897-906.

Belokurov, V., Zucker, D. B., Evans, N. W., Wilkinson, M. I., Irwin, M. J., Hodgkin, S., Bramich, D. M., Irwin, J. M., Gilmore, G., Willman, B., Vidrih, S., Newberg, H. J., Wyse, R. F. G., Fellhauer, M., Hewett, P. C., Cole, N., Bell, E. F., Beers, T. C., Rockosi, C. M., Yanny, B., Grebel, E. K., Schneider, D. P., Lupton, R., Barentine, J. C., Brewington, H., Brinkmann, J., Harvanek, M., Kleinman, S. J., Krzesinski, J., Long, D., Nitta, A., Smith, J. A., Snedden, S. A., Aug. 2006c. A Faint New Milky Way Satellite in Bootes. ApJL 647, L111-L114.

Besla, G., Kallivayalil, N., Hernquist, L., van der Marel, R. P., Cox, T. J., Kereš, D., Oct. 2010. Simulations of the Magellanic Stream in a First Infall Scenario. ApJL 721, L97-L101.

Bonaca, A., Geha, M., Kallivayalil, N., Nov. 2012a. A Cold Milky Way Stellar Stream in the Direction of Triangulum. ApJL 760, L6.

Bonaca, A., Jurić, M., Ivezić, Ž., Bizyaev, D., Brewington, H., Malanushenko, E., Malanushenko, V., Oravetz, D., Pan, K., Shelden, A., 
Simmons, A., Snedden, S., May 2012b. Update on the Nature of Virgo Overdensity. AJ 143, 105.

Bond, N. A., Ivezić, Ž., Sesar, B., Jurić, M., Munn, J. A., Kowalski, A., Loebman, S., Roškar, R., Beers, T. C., Dalcanton, J., Rockosi, C. M., Yanny, B., Newberg, H. J., Allende Prieto, C., Wilhelm, R., Lee, Y. S., Sivarani, T., Majewski, S. R., Norris, J. E., Bailer-Jones, C. A. L., Re Fiorentin, P., Schlegel, D., Uomoto, A., Lupton, R. H., Knapp, G. R., Gunn, J. E., Covey, K. R., Allyn Smith, J., Miknaitis, G., Doi, M., Tanaka, M., Fukugita, M., Kent, S., Finkbeiner, D., Quinn, T. R., Hawley, S., Anderson, S., Kiuchi, F., Chen, A., Bushong, J., Sohi, H., Haggard, D., Kimball, A., McGurk, R., Barentine, J., Brewington, H., Harvanek, M., Kleinman, S., Krzesinski, J., Long, D., Nitta, A., Snedden, S., Lee, B., Pier, J. R., Harris, H., Brinkmann, J., Schneider, D. P., Jun. 2010. The Milky Way Tomography with SDSS. III. Stellar Kinematics. ApJ 716, 129.

Bovy, J., Allende Prieto, C., Beers, T. C., Bizyaev, D., da Costa, L. N., Cunha, K., Ebelke, G. L., Eisenstein, D. J., Frinchaboy, P. M., García Pérez, A. E., Girardi, L., Hearty, F. R., Hogg, D. W., Holtzman, J., Maia, M. A. G., Majewski, S. R., Malanushenko, E., Malanushenko, V., Mészáros, S., Nidever, D. L., O'Connell, R. W., O'Donnell, C., Oravetz, A., Pan, K., Rocha-Pinto, H. J., Schiavon, R. P., Schneider, D. P., Schultheis, M., Skrutskie, M., Smith, V. V., Weinberg, D. H., Wilson, J. C., Zasowski, G., Nov. 2012. The Milky Way's Circular-velocity Curve between 4 and 14 kpc from APOGEE data. ApJ 759, 131.

Boylan-Kolchin, M., Bullock, J. S., Kaplinghat, M., May 2012. The Milky Way's bright satellites as an apparent failure of $\Lambda$ CDM. MNRAS 422, 1203-1218.

Boylan-Kolchin, M., Springel, V., White, S. D. M., Jenkins, A., Aug. 2010. There's no place like home? Statistics of Milky Way-mass dark matter haloes. MNRAS 406, 896-912.

Bramich, D. M., Vidrih, S., Wyrzykowski, L., Munn, J. A., Lin, H., Evans, N. W., Smith, M. C., Belokurov, V., Gilmore, G., Zucker, D. B., Hewett, P. C., Watkins, L. L., Faria, D. C., Fellhauer, M., Miknaitis, G., Bizyaev, D., Ivezić, Ž., Schneider, D. P., Snedden, S. A., Malanushenko, E., 
Malanushenko, V., Pan, K., May 2008. Light and motion in SDSS Stripe 82: the catalogues. MNRAS 386, 887-902.

Brooks, A. M., Kuhlen, M., Zolotov, A., Hooper, D., Mar. 2013. A Baryonic Solution to the Missing Satellites Problem. ApJ 765, 22.

Brown, T. M., Tumlinson, J., Geha, M., Kirby, E. N., VandenBerg, D. A., Muñoz, R. R., Kalirai, J. S., Simon, J. D., Avila, R. J., Guhathakurta, P., Renzini, A., Ferguson, H. C., Jul. 2012. The Primeval Populations of the Ultra-faint Dwarf Galaxies. ApJL 753, L21.

Bullock, J. S., Johnston, K. V., Dec. 2005. Tracing Galaxy Formation with Stellar Halos. I. Methods. ApJ 635, 931-949.

Burnett, B., Binney, J., Sharma, S., Williams, M., Zwitter, T., Bienaymé, O., Bland-Hawthorn, J., Freeman, K. C., Fulbright, J., Gibson, B., Gilmore, G., Grebel, E. K., Helmi, A., Munari, U., Navarro, J. F., Parker, Q. A., Seabroke, G. M., Siebert, A., Siviero, A., Steinmetz, M., Watson, F. G., Wyse, R. F. G., Aug. 2011. Distance determination for RAVE stars using stellar models. III. The nature of the RAVE survey and Milky Way chemistry. A\&A 532, A113.

Busha, M. T., Marshall, P. J., Wechsler, R. H., Klypin, A., Primack, J., Dec. 2011a. The Mass Distribution and Assembly of the Milky Way from the Properties of the Magellanic Clouds. ApJ 743, 40.

Busha, M. T., Wechsler, R. H., Behroozi, P. S., Gerke, B. F., Klypin, A. A., Primack, J. R., Dec. 2011b. Statistics of Satellite Galaxies around MilkyWay-like Hosts. ApJ 743, 117.

Carlin, J. L., Grillmair, C. J., Muñoz, R. R., Nidever, D. L., Majewski, S. R., Sep. 2009. Kinematics and Metallicities in the Boötes III Stellar Overdensity: A Disrupted Dwarf Galaxy? ApJL 702, L9-L13.

Carlin, J. L., Majewski, S. R., Casetti-Dinescu, D. I., Law, D. R., Girard, T. M., Patterson, R. J., Jan. 2012. Kinematics and Chemistry of Stars along the Sagittarius Trailing Tidal Tail and Constraints on the Milky Way Mass Distribution. ApJ 744, 25. 
Casas, R. A., Arias, V., Peña Ramírez, K., Kroupa, P., Aug. 2012. Dwarf spheroidal satellites of the Milky Way from dark matter free tidal dwarf galaxy progenitors: maps of orbits. MNRAS 424, 1941-1951.

Chou, M.-Y., Cunha, K., Majewski, S. R., Smith, V. V., Patterson, R. J., Martínez-Delgado, D., Geisler, D., Jan. 2010. A Two Micron All Sky Survey View of the Sagittarius Dwarf Galaxy. VI. s-Process and Titanium Abundance Variations Along the Sagittarius Stream. ApJ 708, 1290-1309.

Chou, M.-Y., Majewski, S. R., Cunha, K., Smith, V. V., Patterson, R. J., Martínez-Delgado, D., Law, D. R., Crane, J. D., Muñoz, R. R., Garcia López, R., Geisler, D., Skrutskie, M. F., Nov. 2007. A 2MASS All-Sky View of the Sagittarius Dwarf Galaxy. V. Variation of the Metallicity Distribution Function along the Sagittarius Stream. ApJ 670, 346-362.

Conn, B. C., Noël, N. E. D., Rix, H.-W., Lane, R. R., Lewis, G. F., Irwin, M. J., Martin, N. F., Ibata, R. A., Dolphin, A., Chapman, S., Aug. 2012. Slicing the Monoceros Overdensity with SUPRIME-CAM. ApJ 754, 101.

Cooper, A. P., Cole, S., Frenk, C. S., White, S. D. M., Helly, J., Benson, A. J., De Lucia, G., Helmi, A., Jenkins, A., Navarro, J. F., Springel, V., Wang, J., Aug. 2010. Galactic stellar haloes in the CDM model. MNRAS 406, 744-766.

de Jong, J. T. A., Yanny, B., Rix, H.-W., Dolphin, A. E., Martin, N. F., Beers, T. C., May 2010. Mapping the Stellar Structure of the Milky Way Thick Disk and Halo Using SEGUE Photometry. ApJ 714, 663-674.

Deason, A. J., Belokurov, V., Evans, N. W., Oct. 2011. The Milky Way stellar halo out to $40 \mathrm{kpc}$ : squashed, broken but smooth. MNRAS 416, 2903-2915.

Deason, A. J., Belokurov, V., Evans, N. W., An, J., Jul. 2012a. Broken degeneracies: the rotation curve and velocity anisotropy of the Milky Way halo. MNRAS 424, L44-L48.

Deason, A. J., Belokurov, V., Evans, N. W., Johnston, K. V., Feb. 2013. Broken and Unbroken: The Milky Way and M31 Stellar Halos. ApJ 763, 113. 
Deason, A. J., Belokurov, V., Evans, N. W., Koposov, S. E., Cooke, R. J., Peñarrubia, J., Laporte, C. F. P., Fellhauer, M., Walker, M. G., Olszewski, E. W., Oct. 2012b. The cold veil of the Milky Way stellar halo. MNRAS $425,2840-2853$.

Diemand, J., Kuhlen, M., Madau, P., Zemp, M., Moore, B., Potter, D., Stadel, J., Aug. 2008. Clumps and streams in the local dark matter distribution. Nature 454, 735-738.

D'Onghia, E., Springel, V., Hernquist, L., Keres, D., Feb. 2010. Substructure Depletion in the Milky Way Halo by the Disk. ApJ 709, 1138-1147.

Eggen, O. J., Lynden-Bell, D., Sandage, A. R., Nov. 1962. Evidence from the motions of old stars that the Galaxy collapsed. ApJ 136, 748.

Eyre, A., Binney, J., May 2011. The mechanics of tidal streams. MNRAS $413,1852-1874$.

Fellhauer, M., Evans, N. W., Belokurov, V., Zucker, D. B., Yanny, B., Wilkinson, M. I., Gilmore, G., Irwin, M. J., Bramich, D. M., Vidrih, S., Hewett, P., Beers, T., Mar. 2007. Is Ursa Major II the progenitor of the Orphan Stream? MNRAS 375, 1171-1179.

Forero-Romero, J. E., Hoffman, Y., Yepes, G., Gottlöber, S., Piontek, R., Klypin, A., Steinmetz, M., Oct. 2011. The dark matter assembly of the Local Group in constrained cosmological simulations of a $\Lambda$ cold dark matter universe. MNRAS 417, 1434-1443.

Frebel, A., Bromm, V., Nov. 2012. Chemical Signatures of the First Galaxies: Criteria for One-shot Enrichment. ApJ 759, 115.

Frebel, A., Simon, J. D., Geha, M., Willman, B., Jan. 2010. High-Resolution Spectroscopy of Extremely Metal-Poor Stars in the Least Evolved Galaxies: Ursa Major II and Coma Berenices. ApJ 708, 560-583.

Garrison-Kimmel, S., Rocha, M., Boylan-Kolchin, M., Bullock, J., Lally, J., Jan. 2013. Can Feedback Solve the Too Big to Fail Problem? ArXiv e-prints.

Gieles, M., Heggie, D. C., Zhao, H., Jun. 2011. The life cycle of star clusters in a tidal field. MNRAS 413, 2509-2524. 
Gilbert, K. M., Guhathakurta, P., Beaton, R. L., Bullock, J., Geha, M. C., Kalirai, J. S., Kirby, E. N., Majewski, S. R., Ostheimer, J. C., Patterson, R. J., Tollerud, E. J., Tanaka, M., Chiba, M., Nov. 2012. Global Properties of M31's Stellar Halo from the SPLASH Survey. I. Surface Brightness Profile. ApJ 760, 76.

Gilmore, G., Norris, J. E., Monaco, L., Yong, D., Wyse, R. F. G., Geisler, D., Jan. 2013. Elemental Abundances and their Implications for the Chemical Enrichment of the Boötes I Ultrafaint Galaxy. ApJ 763, 61.

Grillmair, C. J., Jul. 2006a. Detection of a $60^{\circ}$-long Dwarf Galaxy Debris Stream. ApJL 645, L37-L40.

Grillmair, C. J., Nov. 2006b. Substructure in Tidal Streams: Tributaries in the Anticenter Stream. ApJL 651, L29-L32.

Grillmair, C. J., Mar. 2009. Four New Stellar Debris Streams in the Galactic Halo. ApJ 693, 1118-1127.

Grillmair, C. J., Sep. 2011. Another Look at the Eastern Banded Structure: A Stellar Debris Stream and a Possible Progenitor. ApJ 738, 98.

Grillmair, C. J., Carlin, J. L., Majewski, S. R., Dec. 2008. Fishing in Tidal Streams: New Radial Velocity and Proper Motion Constraints on the Orbit of the Anticenter Stream. ApJL 689, L117-L120.

Grillmair, C. J., Dionatos, O., Apr. 2006a. A $22^{\circ}$ Tidal Tail for Palomar 5. ApJL 641, L37-L39.

Grillmair, C. J., Dionatos, O., May 2006b. Detection of a $63^{\circ}$ Cold Stellar Stream in the Sloan Digital Sky Survey. ApJL 643, L17-L20.

Grillmair, C. J., Freeman, K. C., Irwin, M., Quinn, P. J., Jun. 1995. Globular Clusters with Tidal Tails: Deep Two-Color Star Counts. AJ 109, 2553.

Hammer, F., Puech, M., Chemin, L., Flores, H., Lehnert, M. D., Jun. 2007. The Milky Way, an Exceptionally Quiet Galaxy: Implications for the Formation of Spiral Galaxies. ApJ 662, 322-334. 
Hammer, F., Yang, Y., Fouquet, S., Pawlowski, M. S., Kroupa, P., Puech, M., Flores, H., Wang, J., Mar. 2013. The vast thin plane of M31 corotating dwarfs: an additional fossil signature of the M31 merger and of its considerable impact in the whole Local Group. ArXiv e-prints.

Helmi, A., Jun. 2008. The stellar halo of the Galaxy. A\&AR 15, 145-188.

Helmi, A., Cooper, A. P., White, S. D. M., Cole, S., Frenk, C. S., Navarro, J. F., May 2011. Substructure in the Stellar Halos of the Aquarius Simulations. ApJL 733, L7.

Helmi, A., White, S. D. M., de Zeeuw, P. T., Zhao, H., Nov. 1999. Debris streams in the solar neighbourhood as relicts from the formation of the Milky Way. Nature 402, 53-55.

Ibata, R., Bellazzini, M., Chapman, S. C., Dalessandro, E., Ferraro, F., Irwin, M., Lanzoni, B., Lewis, G. F., Mackey, A. D., Miocchi, P., Varghese, A., Jul. 2009. Density and Kinematic Cusps in M54 at the Heart of the Sagittarius Dwarf Galaxy: Evidence for A $10^{4} \mathrm{M}$ sun Black Hole? ApJL 699, L169-L173.

Ibata, R. A., Gilmore, G., Irwin, M. J., Jul. 1994. A dwarf satellite galaxy in Sagittarius. Nature 370, 194-196.

Ibata, R. A., Irwin, M. J., Lewis, G. F., Ferguson, A. M. N., Tanvir, N., Apr. 2003. One ring to encompass them all: a giant stellar structure that surrounds the Galaxy. MNRAS 340, L21-L27.

Ibata, R. A., Lewis, G. F., Conn, A. R., Irwin, M. J., McConnachie, A. W., Chapman, S. C., Collins, M. L., Fardal, M., Ferguson, A. M. N., Ibata, N. G., Mackey, A. D., Martin, N. F., Navarro, J., Rich, R. M., VallsGabaud, D., Widrow, L. M., Jan. 2013. A vast, thin plane of corotating dwarf galaxies orbiting the Andromeda galaxy. Nature 493, 62-65.

Irwin, M. J., Jan. 1994. Searches for Galactic Dwarf Spheroidal Satellites. In: Meylan, G., Prugniel, P. (Eds.), European Southern Observatory Conference and Workshop Proceedings. Vol. 49 of European Southern Observatory Conference and Workshop Proceedings. p. 27.

Irwin, M. J., Belokurov, V., Evans, N. W., Ryan-Weber, E. V., de Jong, J. T. A., Koposov, S., Zucker, D. B., Hodgkin, S. T., Gilmore, G., Prema, 
P., Hebb, L., Begum, A., Fellhauer, M., Hewett, P. C., Kennicutt, Jr., R. C., Wilkinson, M. I., Bramich, D. M., Vidrih, S., Rix, H.-W., Beers, T. C., Barentine, J. C., Brewington, H., Harvanek, M., Krzesinski, J., Long, D., Nitta, A., Snedden, S. A., Feb. 2007. Discovery of an Unusual Dwarf Galaxy in the Outskirts of the Milky Way. ApJL 656, L13-L16.

Ivezić, Ž., Beers, T. C., Jurić, M., Sep. 2012. Galactic Stellar Populations in the Era of the Sloan Digital Sky Survey and Other Large Surveys. ARA\&A 50, 251-304.

Ivezić, Ž., Sesar, B., Jurić, M., Bond, N., Dalcanton, J., Rockosi, C. M., Yanny, B., Newberg, H. J., Beers, T. C., Allende Prieto, C., Wilhelm, R., Lee, Y. S., Sivarani, T., Norris, J. E., Bailer-Jones, C. A. L., Re Fiorentin, P., Schlegel, D., Uomoto, A., Lupton, R. H., Knapp, G. R., Gunn, J. E., Covey, K. R., Smith, J. A., Miknaitis, G., Doi, M., Tanaka, M., Fukugita, M., Kent, S., Finkbeiner, D., Munn, J. A., Pier, J. R., Quinn, T., Hawley, S., Anderson, S., Kiuchi, F., Chen, A., Bushong, J., Sohi, H., Haggard, D., Kimball, A., Barentine, J., Brewington, H., Harvanek, M., Kleinman, S., Krzesinski, J., Long, D., Nitta, A., Snedden, S., Lee, B., Harris, H., Brinkmann, J., Schneider, D. P., York, D. G., Sep. 2008. The Milky Way Tomography with SDSS. II. Stellar Metallicity. ApJ 684, 287-325.

Jurić, M., Ivezić, Ž., Brooks, A., Lupton, R. H., Schlegel, D., Finkbeiner, D., Padmanabhan, N., Bond, N., Sesar, B., Rockosi, C. M., Knapp, G. R., Gunn, J. E., Sumi, T., Schneider, D. P., Barentine, J. C., Brewington, H. J., Brinkmann, J., Fukugita, M., Harvanek, M., Kleinman, S. J., Krzesinski, J., Long, D., Neilsen, Jr., E. H., Nitta, A., Snedden, S. A., York, D. G., Feb. 2008. The Milky Way Tomography with SDSS. I. Stellar Number Density Distribution. ApJ 673, 864-914.

Keller, S. C., Yong, D., Da Costa, G. S., Sep. 2010. The Chemistry of the Trailing Arm of the Sagittarius Dwarf Galaxy. ApJ 720, 940-947.

Kirby, E. N., Boylan-Kolchin, M., Cohen, J. G., Geha, M., Bullock, J. S., Kaplinghat, M., Apr. 2013. Segue 2: The Least Massive Galaxy. ArXiv e-prints.

Kirby, E. N., Simon, J. D., Geha, M., Guhathakurta, P., Frebel, A., Sep. 2008. Uncovering Extremely Metal-Poor Stars in the Milky Way's Ultrafaint Dwarf Spheroidal Satellite Galaxies. ApJL 685, L43-L46. 
Kleyna, J. T., Geller, M. J., Kenyon, S. J., Kurtz, M. J., Feb. 1997. An Adaptive Kernel Approach to Finding dSph Galaxies Around the Milky Way. AJ 113, 624-633.

Klypin, A., Kravtsov, A. V., Valenzuela, O., Prada, F., Sep. 1999. Where Are the Missing Galactic Satellites? ApJ 522, 82-92.

Koch, A., Feltzing, S., Adén, D., Matteucci, F., Jun. 2013. Neutron-capture element deficiency of the Hercules dwarf spheroidal galaxy. A\&A 554, A5.

Koch, A., McWilliam, A., Grebel, E. K., Zucker, D. B., Belokurov, V., Nov. 2008. The Highly Unusual Chemical Composition of the Hercules Dwarf Spheroidal Galaxy. ApJL 688, L13-L16.

Kollmeier, J. A., Gould, A., Shectman, S., Thompson, I. B., Preston, G. W., Simon, J. D., Crane, J. D., Ivezić, Ž., Sesar, B., Nov. 2009. Spectroscopic Confirmation of the Pisces Overdensity. ApJL 705, L158-L162.

Koposov, S., Belokurov, V., Evans, N. W., Hewett, P. C., Irwin, M. J., Gilmore, G., Zucker, D. B., Rix, H.-W., Fellhauer, M., Bell, E. F., Glushkova, E. V., Oct. 2008. The Luminosity Function of the Milky Way Satellites. ApJ 686, 279-291.

Koposov, S., de Jong, J. T. A., Belokurov, V., Rix, H.-W., Zucker, D. B., Evans, N. W., Gilmore, G., Irwin, M. J., Bell, E. F., Nov. 2007. The Discovery of Two Extremely Low Luminosity Milky Way Globular Clusters. ApJ 669, 337-342.

Koposov, S. E., Belokurov, V., Evans, N. W., Gilmore, G., Gieles, M., Irwin, M. J., Lewis, G. F., Niederste-Ostholt, M., Peñarrubia, J., Smith, M. C., Bizyaev, D., Malanushenko, E., Malanushenko, V., Schneider, D. P., Wyse, R. F. G., May 2012. The Sagittarius Streams in the Southern Galactic Hemisphere. ApJ 750, 80.

Koposov, S. E., Gilmore, G., Walker, M. G., Belokurov, V., Wyn Evans, N., Fellhauer, M., Gieren, W., Geisler, D., Monaco, L., Norris, J. E., Okamoto, S., Peñarrubia, J., Wilkinson, M., Wyse, R. F. G., Zucker, D. B., Aug. 2011. Accurate Stellar Kinematics at Faint Magnitudes: Application to the Boötes I Dwarf Spheroidal Galaxy. ApJ 736, 146. 
Koposov, S. E., Rix, H.-W., Hogg, D. W., Mar. 2010. Constraining the Milky Way Potential with a Six-Dimensional Phase-Space Map of the GD-1 Stellar Stream. ApJ 712, 260-273.

Koposov, S. E., Yoo, J., Rix, H.-W., Weinberg, D. H., Macciò, A. V., Escudé, J. M., May 2009. A Quantitative Explanation of the Observed Population of Milky Way Satellite Galaxies. ApJ 696, 2179-2194.

Kravtsov, A., 2010. Dark Matter Substructure and Dwarf Galactic Satellites. Advances in Astronomy 2010.

Kroupa, P., Jul. 1997. Dwarf spheroidal satellite galaxies without dark matter. New A. 2, 139-164.

Kroupa, P., Theis, C., Boily, C. M., Feb. 2005. The great disk of Milky-Way satellites and cosmological sub-structures. A\&A 431, 517-521.

Lai, D. K., Lee, Y. S., Bolte, M., Lucatello, S., Beers, T. C., Johnson, J. A., Sivarani, T., Rockosi, C. M., Sep. 2011. The $[\mathrm{Fe} / \mathrm{H}],[\mathrm{C} / \mathrm{Fe}]$, and $[\alpha / \mathrm{Fe}]$ Distributions of the Boötes I Dwarf Spheroidal Galaxy. ApJ 738, 51.

Larsen, J. A., Cabanela, J. E., Humphreys, R. M., Apr. 2011. Mapping the Asymmetric Thick Disk. II. Distance, Size, and Mass of the Hercules Thick Disk Cloud. AJ 141, 130.

Larsen, J. A., Humphreys, R. M., Cabanela, J. E., Nov. 2008. Mapping the Asymmetric Thick Disk: The Hercules Thick-Disk Cloud. ApJL 687, L17L19.

Lauchner, A., Powell, Jr., W. L., Wilhelm, R., Nov. 2006. Discovery of a Tidal Stream Extending from NGC 5053. ApJL 651, L33-L36.

Li, J., Newberg, H. J., Carlin, J. L., Deng, L., Newby, M., Willett, B. A., Xu, Y., Luo, Z., Oct. 2012. On Rings and Streams in the Galactic Anti-Center. ApJ 757, 151.

Libeskind, N. I., Frenk, C. S., Cole, S., Helly, J. C., Jenkins, A., Navarro, J. F., Power, C., Oct. 2005. The distribution of satellite galaxies: the great pancake. MNRAS 363, 146-152.

Lynden-Bell, D., Mar. 1976. Dwarf galaxies and globular clusters in high velocity hydrogen streams. MNRAS 174, 695-710. 
Macciò, A. V., Kang, X., Fontanot, F., Somerville, R. S., Koposov, S., Monaco, P., Mar. 2010. Luminosity function and radial distribution of Milky Way satellites in a $\Lambda$ CDM Universe. MNRAS 402, 1995-2008.

Majewski, S. R., Ostheimer, J. C., Rocha-Pinto, H. J., Patterson, R. J., Guhathakurta, P., Reitzel, D., Nov. 2004. Detection of the MainSequence Turnoff of a Newly Discovered Milky Way Halo Structure in the Triangulum-Andromeda Region. ApJ 615, 738-743.

Martin, C., Carlin, J. L., Newberg, H. J., Grillmair, C., Mar. 2013. Kinematic Discovery of a Stellar Stream Located in Pisces. ApJL 765, L39.

Martin, N. F., de Jong, J. T. A., Rix, H.-W., Sep. 2008. A Comprehensive Maximum Likelihood Analysis of the Structural Properties of Faint Milky Way Satellites. ApJ 684, 1075-1092.

Martin, N. F., Ibata, R. A., Chapman, S. C., Irwin, M., Lewis, G. F., Sep. 2007a. A Keck/DEIMOS spectroscopic survey of faint Galactic satellites: searching for the least massive dwarf galaxies. MNRAS 380, 281-300.

Martin, N. F., Ibata, R. A., Irwin, M., Oct. 2007b. Galactic Halo Stellar Structures in the Triangulum-Andromeda Region. ApJL 668, L123-L126.

Martínez-Delgado, D., Gabany, R. J., Crawford, K., Zibetti, S., Majewski, S. R., Rix, H.-W., Fliri, J., Carballo-Bello, J. A., Bardalez-Gagliuffi, D. C., Peñarrubia, J., Chonis, T. S., Madore, B., Trujillo, I., Schirmer, M., McDavid, D. A., Oct. 2010. Stellar Tidal Streams in Spiral Galaxies of the Local Volume: A Pilot Survey with Modest Aperture Telescopes. AJ 140, 962-967.

Martínez-Delgado, D., Romanowsky, A. J., Gabany, R. J., Annibali, F., Arnold, J. A., Fliri, J., Zibetti, S., van der Marel, R. P., Rix, H.-W., Chonis, T. S., Carballo-Bello, J. A., Aloisi, A., Macciò, A. V., GallegoLaborda, J., Brodie, J. P., Merrifield, M. R., Apr. 2012. Dwarfs Gobbling Dwarfs: A Stellar Tidal Stream around NGC 4449 and Hierarchical Galaxy Formation on Small Scales. ApJL 748, L24.

McConnachie, A. W., Jul. 2012. The Observed Properties of Dwarf Galaxies in and around the Local Group. AJ 144, 4. 
Metz, M., Kroupa, P., Mar. 2007. Dwarf spheroidal satellites: are they of tidal origin? MNRAS 376, 387-392.

Moore, B., Ghigna, S., Governato, F., Lake, G., Quinn, T., Stadel, J., Tozzi, P., Oct. 1999. Dark Matter Substructure within Galactic Halos. ApJL 524, L19-L22.

Morrison, H. L., Mateo, M., Olszewski, E. W., Harding, P., Dohm-Palmer, R. C., Freeman, K. C., Norris, J. E., Morita, M., May 2000. Mapping the Galactic Halo. I. The "Spaghetti" Survey. AJ 119, 2254-2273.

Muñoz, R. R., Geha, M., Willman, B., Jul. 2010. Turning the Tides on the Ultra-faint Dwarf Spheroidal Galaxies: Coma Berenices and Ursa Major II. AJ 140, 138-151.

Newberg, H. J., Willett, B. A., Yanny, B., Xu, Y., Mar. 2010. The Orbit of the Orphan Stream. ApJ 711, 32-49.

Newberg, H. J., Yanny, B., Grebel, E. K., Hennessy, G., Ivezić, Ž., MartinezDelgado, D., Odenkirchen, M., Rix, H.-W., Brinkmann, J., Lamb, D. Q., Schneider, D. P., York, D. G., Oct. 2003. Sagittarius Tidal Debris 90 Kiloparsecs from the Galactic Center. ApJL 596, L191-L194.

Newberg, H. J., Yanny, B., Rockosi, C., Grebel, E. K., Rix, H.-W., Brinkmann, J., Csabai, I., Hennessy, G., Hindsley, R. B., Ibata, R., Ivezić, Z., Lamb, D., Nash, E. T., Odenkirchen, M., Rave, H. A., Schneider, D. P., Smith, J. A., Stolte, A., York, D. G., Apr. 2002. The Ghost of Sagittarius and Lumps in the Halo of the Milky Way. ApJ 569, 245-274.

Newberg, H. J., Yanny, B., Willett, B. A., Aug. 2009. Discovery of a New, Polar-Orbiting Debris Stream in the Milky Way Stellar Halo. ApJL 700, L61-L64.

Niederste-Ostholt, M., Belokurov, V., Evans, N. W., Koposov, S., Gieles, M., Irwin, M. J., Oct. 2010a. The tidal tails of the ultrafaint globular cluster Palomar 1. MNRAS 408, L66-L70.

Niederste-Ostholt, M., Belokurov, V., Evans, N. W., Peñarrubia, J., Mar. 2010b. Re-Assembling the Sagittarius Dwarf Galaxy. ApJ 712, 516-526. 
Norris, J. E., Wyse, R. F. G., Gilmore, G., Yong, D., Frebel, A., Wilkinson, M. I., Belokurov, V., Zucker, D. B., Nov. 2010. Chemical Enrichment in the Faintest Galaxies: The Carbon and Iron Abundance Spreads in the Boötes I Dwarf Spheroidal Galaxy and the Segue 1 System. ApJ 723, 1632-1650.

Odenkirchen, M., Grebel, E. K., Rockosi, C. M., Dehnen, W., Ibata, R., Rix, H.-W., Stolte, A., Wolf, C., Anderson, Jr., J. E., Bahcall, N. A., Brinkmann, J., Csabai, I., Hennessy, G., Hindsley, R. B., Ivezić, Ž., Lupton, R. H., Munn, J. A., Pier, J. R., Stoughton, C., York, D. G., Feb. 2001. Detection of Massive Tidal Tails around the Globular Cluster Palomar 5 with Sloan Digital Sky Survey Commissioning Data. ApJL 548, L165-L169.

Okamoto, S., Arimoto, N., Yamada, Y., Onodera, M., Jan. 2012. Stellar Populations and Structural Properties of Ultra Faint Dwarf Galaxies, Canes Venatici I, Boötes I, Canes Venatici II, and Leo IV. ApJ 744, 96.

Palma, C., Majewski, S. R., Johnston, K. V., Jan. 2002. On the Distribution of Orbital Poles of Milky Way Satellites. ApJ 564, 736-761.

Pawlowski, M. S., Pflamm-Altenburg, J., Kroupa, P., Jun. 2012. The VPOS: a vast polar structure of satellite galaxies, globular clusters and streams around the Milky Way. MNRAS 423, 1109-1126.

Peñarrubia, J., Benson, A. J., Walker, M. G., Gilmore, G., McConnachie, A. W., Mayer, L., Aug. 2010. The impact of dark matter cusps and cores on the satellite galaxy population around spiral galaxies. MNRAS 406, 1290-1305.

Peñarrubia, J., Martínez-Delgado, D., Rix, H. W., Gómez-Flechoso, M. A., Munn, J., Newberg, H., Bell, E. F., Yanny, B., Zucker, D., Grebel, E. K., Jun. 2005. A Comprehensive Model for the Monoceros Tidal Stream. ApJ $626,128-144$.

Peñarrubia, J., Pontzen, A., Walker, M. G., Koposov, S. E., Nov. 2012. The Coupling between the Core/Cusp and Missing Satellite Problems. ApJL 759, L42.

Pontzen, A., Governato, F., Apr. 2012. How supernova feedback turns dark matter cusps into cores. MNRAS 421, 3464-3471. 
Purcell, C. W., Zentner, A. R., Dec. 2012. Bailing out the Milky Way: variation in the properties of massive dwarfs among galaxy-sized systems. J. Cosmology Astropart. Phys. 12, 7.

Read, J. I., Wilkinson, M. I., Evans, N. W., Gilmore, G., Kleyna, J. T., Mar. 2006. The importance of tides for the Local Group dwarf spheroidals. MNRAS 367, 387-399.

Reid, M. J., Menten, K. M., Zheng, X. W., Brunthaler, A., Moscadelli, L., Xu, Y., Zhang, B., Sato, M., Honma, M., Hirota, T., Hachisuka, K., Choi, Y. K., Moellenbrock, G. A., Bartkiewicz, A., Jul. 2009. Trigonometric Parallaxes of Massive Star-Forming Regions. VI. Galactic Structure, Fundamental Parameters, and Noncircular Motions. ApJ 700, 137-148.

Rocha-Pinto, H. J., Majewski, S. R., Skrutskie, M. F., Crane, J. D., Sep. 2003. Tracing the Galactic Anticenter Stellar Stream with 2MASS M Giants. ApJL 594, L115-L118.

Rocha-Pinto, H. J., Majewski, S. R., Skrutskie, M. F., Crane, J. D., Patterson, R. J., Nov. 2004. Exploring Halo Substructure with Giant Stars: A Diffuse Star Cloud or Tidal Debris around the Milky Way in TriangulumAndromeda. ApJ 615, 732-737.

Rockosi, C. M., Odenkirchen, M., Grebel, E. K., Dehnen, W., Cudworth, K. M., Gunn, J. E., York, D. G., Brinkmann, J., Hennessy, G. S., Ivezić, Ž., Jul. 2002. A Matched-Filter Analysis of the Tidal Tails of the Globular Cluster Palomar 5. AJ 124, 349-363.

Sand, D. J., Olszewski, E. W., Willman, B., Zaritsky, D., Seth, A., Harris, J., Piatek, S., Saha, A., Oct. 2009. The Star Formation History and Extended Structure of the Hercules Milky Way Satellite. ApJ 704, 898-914.

Sand, D. J., Strader, J., Willman, B., Zaritsky, D., McLeod, B., Caldwell, N., Seth, A., Olszewski, E., Sep. 2012. Tidal Signatures in the Faintest Milky Way Satellites: The Detailed Properties of Leo V, Pisces II, and Canes Venatici II. ApJ 756, 79.

Sanders, J. L., Binney, J., May 2013. Stream-orbit misalignment II: A new algorithm to constrain the Galactic potential. ArXiv e-prints. 
Schlaufman, K. C., Rockosi, C. M., Allende Prieto, C., Beers, T. C., Bizyaev, D., Brewington, H., Lee, Y. S., Malanushenko, V., Malanushenko, E., Oravetz, D., Pan, K., Simmons, A., Snedden, S., Yanny, B., Oct. 2009. Insight into the Formation of the Milky Way Through Cold Halo Substructure. I. The ECHOS of Milky Way Formation. ApJ 703, 2177-2204.

Sesar, B., Ivezić, Ž., Grammer, S. H., Morgan, D. P., Becker, A. C., Jurić, M., De Lee, N., Annis, J., Beers, T. C., Fan, X., Lupton, R. H., Gunn, J. E., Knapp, G. R., Jiang, L., Jester, S., Johnston, D. E., Lampeitl, H., Jan. 2010a. Light Curve Templates and Galactic Distribution of RR Lyrae Stars from Sloan Digital Sky Survey Stripe 82. ApJ 708, 717-741.

Sesar, B., Vivas, A. K., Duffau, S., Ivezić, Ž., Jul. 2010b. Halo Velocity Groups in the Pisces Overdensity. ApJ 717, 133-139.

Simon, J. D., Frebel, A., McWilliam, A., Kirby, E. N., Thompson, I. B., Jun. 2010. High-resolution Spectroscopy of Extremely Metal-poor Stars in the Least Evolved Galaxies: Leo IV. ApJ 716, 446-452.

Simon, J. D., Geha, M., Nov. 2007. The Kinematics of the Ultra-faint Milky Way Satellites: Solving the Missing Satellite Problem. ApJ 670, 313-331.

Simon, J. D., Geha, M., Minor, Q. E., Martinez, G. D., Kirby, E. N., Bullock, J. S., Kaplinghat, M., Strigari, L. E., Willman, B., Choi, P. I., Tollerud, E. J., Wolf, J., May 2011. A Complete Spectroscopic Survey of the Milky Way Satellite Segue 1: The Darkest Galaxy. ApJ 733, 46.

Smith, M. C., Evans, N. W., Belokurov, V., Hewett, P. C., Bramich, D. M., Gilmore, G., Irwin, M. J., Vidrih, S., Zucker, D. B., Nov. 2009. Kinematics of SDSS subdwarfs: structure and substructure of the Milky Way halo. MNRAS 399, 1223-1237.

Smith, M. C., Ruchti, G. R., Helmi, A., Wyse, R. F. G., Fulbright, J. P., Freeman, K. C., Navarro, J. F., Seabroke, G. M., Steinmetz, M., Williams, M., Bienaymé, O., Binney, J., Bland-Hawthorn, J., Dehnen, W., Gibson, B. K., Gilmore, G., Grebel, E. K., Munari, U., Parker, Q. A., Scholz, R.D., Siebert, A., Watson, F. G., Zwitter, T., Aug. 2007. The RAVE survey: constraining the local Galactic escape speed. MNRAS 379, 755-772.

Smith, M. C., Whiteoak, S. H., Evans, N. W., Feb. 2012. Slicing and Dicing the Milky Way Disk in the Sloan Digital Sky Survey. ApJ 746, 181. 
Sollima, A., Martínez-Delgado, D., Valls-Gabaud, D., Peñarrubia, J., Jan. 2011. Discovery of Tidal Tails Around the Distant Globular Cluster Palomar 14. ApJ 726, 47.

Springel, V., Wang, J., Vogelsberger, M., Ludlow, A., Jenkins, A., Helmi, A., Navarro, J. F., Frenk, C. S., White, S. D. M., Dec. 2008. The Aquarius Project: the subhaloes of galactic haloes. MNRAS 391, 1685-1711.

Stadel, J., Potter, D., Moore, B., Diemand, J., Madau, P., Zemp, M., Kuhlen, M., Quilis, V., Sep. 2009. Quantifying the heart of darkness with GHALO a multibillion particle simulation of a galactic halo. MNRAS 398, L21-L25.

Starkenburg, E., Helmi, A., Morrison, H. L., Harding, P., van Woerden, H., Mateo, M., Olszewski, E. W., Sivarani, T., Norris, J. E., Freeman, K. C., Shectman, S. A., Dohm-Palmer, R. C., Frey, L., Oravetz, D., Jun. 2009. Mapping the Galactic Halo. VIII. Quantifying Substructure. ApJ $698,567-579$.

Taylor, J. E., Babul, A., Oct. 2001. The Dynamics of Sinking Satellites around Disk Galaxies: A Poor Man's Alternative to High-Resolution Numerical Simulations. ApJ 559, 716-735.

Tollerud, E. J., Bullock, J. S., Strigari, L. E., Willman, B., Nov. 2008. Hundreds of Milky Way Satellites? Luminosity Bias in the Satellite Luminosity Function. ApJ 688, 277-289.

Tolstoy, E., Hill, V., Tosi, M., Sep. 2009. Star-Formation Histories, Abundances, and Kinematics of Dwarf Galaxies in the Local Group. ARA\&A $47,371-425$.

Vargas, L. C., Geha, M., Kirby, E. N., Simon, J. D., Apr. 2013. The Distribution of Alpha Elements in Ultra-faint Dwarf Galaxies. ApJ 767, 134.

Vera-Ciro, C. A., Helmi, A., Starkenburg, E., Breddels, M. A., Jan. 2013. Not too big, not too small: the dark haloes of the dwarf spheroidals in the Milky Way. MNRAS 428, 1696-1703.

Walker, M. G., May 2012. Dark Matter in the Milky Way's Dwarf Spheroidal Satellites. ArXiv e-prints. 
Walker, M. G., Belokurov, V., Evans, N. W., Irwin, M. J., Mateo, M., Olszewski, E. W., Gilmore, G., Apr. 2009. Leo V: Spectroscopy of a Distant and Disturbed Satellite. ApJL 694, L144-L147.

Walsh, S. M., Jerjen, H., Willman, B., Jun. 2007. A Pair of Boötes: A New Milky Way Satellite. ApJL 662, L83-L86.

Watkins, L. L., Evans, N. W., An, J. H., Jul. 2010. The masses of the Milky Way and Andromeda galaxies. MNRAS 406, 264-278.

Watkins, L. L., Evans, N. W., Belokurov, V., Smith, M. C., Hewett, P. C., Bramich, D. M., Gilmore, G. F., Irwin, M. J., Vidrih, S., Wyrzykowski, Ł., Zucker, D. B., Oct. 2009. Substructure revealed by RRLyraes in SDSS Stripe 82. MNRAS 398, 1757-1770.

Wechsler, R. H., Bullock, J. S., Primack, J. R., Kravtsov, A. V., Dekel, A., Mar. 2002. Concentrations of Dark Halos from Their Assembly Histories. ApJ 568, 52-70.

Wechsler, R. H., Zentner, A. R., Bullock, J. S., Kravtsov, A. V., Allgood, B., Nov. 2006. The Dependence of Halo Clustering on Halo Formation History, Concentration, and Occupation. ApJ 652, 71-84.

Willman, B., 2010. In Pursuit of the Least Luminous Galaxies. Advances in Astronomy 2010.

Willman, B., Blanton, M. R., West, A. A., Dalcanton, J. J., Hogg, D. W., Schneider, D. P., Wherry, N., Yanny, B., Brinkmann, J., Jun. 2005a. A New Milky Way Companion: Unusual Globular Cluster or Extreme Dwarf Satellite? AJ 129, 2692-2700.

Willman, B., Dalcanton, J., Ivezić, Ž., Jackson, T., Lupton, R., Brinkmann, J., Hennessy, G., Hindsley, R., Feb. 2002. An SDSS Survey For Resolved Milky Way Satellite Galaxies. I. Detection Limits. AJ 123, 848-854.

Willman, B., Dalcanton, J. J., Martinez-Delgado, D., West, A. A., Blanton, M. R., Hogg, D. W., Barentine, J. C., Brewington, H. J., Harvanek, M., Kleinman, S. J., Krzesinski, J., Long, D., Neilsen, Jr., E. H., Nitta, A., Snedden, S. A., Jun. 2005b. A New Milky Way Dwarf Galaxy in Ursa Major. ApJL 626, L85-L88. 
Willman, B., Geha, M., Strader, J., Strigari, L. E., Simon, J. D., Kirby, E., Ho, N., Warres, A., Oct. 2011. Willman 1. A Probable Dwarf Galaxy with an Irregular Kinematic Distribution. AJ 142, 128.

Xue, X.-X., Rix, H.-W., Yanny, B., Beers, T. C., Bell, E. F., Zhao, G., Bullock, J. S., Johnston, K. V., Morrison, H., Rockosi, C., Koposov, S. E., Kang, X., Liu, C., Luo, A., Lee, Y. S., Weaver, B. A., Sep. 2011. Quantifying Kinematic Substructure in the Milky Way's Stellar Halo. ApJ 738, 79 .

Xue, X. X., Rix, H. W., Zhao, G., Re Fiorentin, P., Naab, T., Steinmetz, M., van den Bosch, F. C., Beers, T. C., Lee, Y. S., Bell, E. F., Rockosi, C., Yanny, B., Newberg, H., Wilhelm, R., Kang, X., Smith, M. C., Schneider, D. P., Sep. 2008. The Milky Way's Circular Velocity Curve to $60 \mathrm{kpc}$ and an Estimate of the Dark Matter Halo Mass from the Kinematics of $\sim 2400$ SDSS Blue Horizontal-Branch Stars. ApJ 684, 1143-1158.

Yanny, B., Newberg, H. J., Grebel, E. K., Kent, S., Odenkirchen, M., Rockosi, C. M., Schlegel, D., Subbarao, M., Brinkmann, J., Fukugita, M., Ivezic, Ž., Lamb, D. Q., Schneider, D. P., York, D. G., May 2003. A Low-Latitude Halo Stream around the Milky Way. ApJ 588, 824-841.

Yanny, B., Newberg, H. J., Johnson, J. A., Lee, Y. S., Beers, T. C., Bizyaev, D., Brewington, H., Fiorentin, P. R., Harding, P., Malanushenko, E., Malanushenko, V., Oravetz, D., Pan, K., Simmons, A., Snedden, S., Aug. 2009. Tracing Sagittarius Structure with SDSS and SEGUE Imaging and Spectroscopy. ApJ 700, 1282-1298.

Yanny, B., Newberg, H. J., Kent, S., Laurent-Muehleisen, S. A., Pier, J. R., Richards, G. T., Stoughton, C., Anderson, Jr., J. E., Annis, J., Brinkmann, J., Chen, B., Csabai, I., Doi, M., Fukugita, M., Hennessy, G. S., Ivezić, Ž., Knapp, G. R., Lupton, R., Munn, J. A., Nash, T., Rockosi, C. M., Schneider, D. P., Smith, J. A., York, D. G., Sep. 2000. Identification of A-colored Stars and Structure in the Halo of the Milky Way from Sloan Digital Sky Survey Commissioning Data. ApJ 540, 825-841.

Zentner, A. R., Berlind, A. A., Bullock, J. S., Kravtsov, A. V., Wechsler, R. H., May 2005a. The Physics of Galaxy Clustering. I. A Model for Subhalo Populations. ApJ 624, 505-525. 